\title{
Renormalization group improved implications of semileptonic operators in SMEFT
}

\author{
Jacky Kumar \\ TUM Institute for Advanced Study, \\ Lichtenbergstr. 2a, D-85747 Garching, Germany \\ E-mail: jacky.kumar@tum.de
}

ABSTRACT: We study implications of the four-fermion semileptonic operators at the lowenergy and at electroweak (EW) scale in the framework of Standard Model Effective Field Theory (SMEFT). We show how the renormalization group (RG) running effects can play an important role in probing the generic flavour structure of such operators. It is shown that at the 1-loop level, through RG running, depending upon the flavour structure, these operators can give rise to sizable effects at low energy in the electroweak precision (EWP) observables, the leptonic, quark, as well as the $Z$ boson flavour violating decays. To this end, we isolate the phenomenologically relevant terms in the full anomalous dimension matrices (ADMs) and discuss the impact of the QED+QCD running in the Weak effective field theory (WET) and the SMEFT running due to gauge and Yukawa interactions on the dim- 4 and dim- 6 operators at the low energy. Considering all the relevant processes, we derive lower bounds on new physics (NP) scale $\Lambda$ for each semileptonic operator, keeping a generic flavour structure. In addition, we also report the allowed ranges for the Wilson coefficients at a fixed value of $\Lambda=3 \mathrm{TeV}$.

Keywords: Beyond Standard Model, Effective Field Theories, Renormalization Group

ArXIV EPRINT: 2107.13005 


\section{Contents}

1 Introduction 1

2 General strategy 3

3 Renormalization group running $\quad 4$

3.1 Evolution of $\Delta F=(0,0)$ operators 5

3.1.1 Operator mixing due to gauge interactions 5

$\begin{array}{lll}3.1 .2 & \text { Operator mixing due to top-Yukawa interactions } & 7\end{array}$

$\begin{array}{ll}3.2 \text { Evolution of } \Delta F=(1,0) \text { operators } & 7\end{array}$

4 Observables induced at 1-loop level $\quad 8$

4.1 Observables for $\Delta F=(0,0)$ operators 8

$\begin{array}{llr}\text { 4.1.1 } & \text { Electroweak precision observables } & 8\end{array}$

$\begin{array}{lll}4.1 .2 & B \text { meson decays } & 11\end{array}$

$\begin{array}{lll}\text { 4.1.3 Charged current decays } & 12\end{array}$

$\begin{array}{lll}\text { 4.1.4 Correlations } & 13\end{array}$

4.2 Observables for $\Delta F=(1,0)$ operators 13

$\begin{array}{lll}4.2 .1 \quad Z \rightarrow \bar{\ell}_{i} \ell_{j} \text { decays } & 14\end{array}$

$\begin{array}{lll}4.2 .2 \tau \rightarrow 3 \ell \text { and } \mu \rightarrow 3 e \text { decays } & 14\end{array}$

4.2.3 $\tau \rightarrow \rho \ell, \tau \rightarrow P(\phi, \pi) \ell$ decays $\quad 15$

$\begin{array}{lll}4.2 .4 & \text { Correlations } & 16\end{array}$

5 Sensitivities to the NP scale $\Lambda \quad 18$

6 Conclusions and outlook 21

A Tree-level shifts in the dim-4 gauge boson couplings 23

B RG induced shifts in the dim-4 and dim-6 operators $\quad 24$

B.1 Dim- $4 Z$ boson couplings 24

B.2 Dim- 4 boson couplings 26

B.3 Leptonic dim-6 WET operators 26

B.4 Semileptonic dim-6 WET operators 27

$\begin{array}{ll}\text { C Bounds on the Wilson coefficients } & 27\end{array}$

\section{Introduction}

In the absence of the discoveries of new particles at the Large Hadron Collider (LHC), the SMEFT provides an elegant framework to parameterize and quantify the effects of $\mathrm{NP}$ in terms of $\mathrm{SU}(3)_{c} \times \mathrm{SU}(2)_{L} \times \mathrm{U}(1)_{Y}$ gauge invariant higher dimensional $(d \geq 5)$ operators $[1,2]$. In SMEFT, the operators are constructed using the field content of the Standard Model (SM). Excluding the flavour structures, there are in total 59 operators which conserve the baryon number [1]. 
An interesting aspect of the SMEFT is its built-in gauge symmetry. This feature often leads to an intriguing pattern of correlations among low energy observables due to enforcement of the model independent relations between the Weak effective theory (WET) operators at the EW scale, on matching with SMEFT [3-6]. At the 1-loop level, through RG running, new effective operators can also be generated at the EW scale as a result of operator mixing. For instance, the four-fermion SMEFT operators can mix with the $\psi^{2} \phi^{2} D$ type operators which can contribute to the observables of different kinds. In this manner, the running from $\Lambda$ to the EW scale can induce additional correlations [7-12]. Therefore, in an SMEFT analysis, considering such effects is very important in order to correctly predict the low energy implications of new interactions introduced at the NP scale.

By now, the complete ADMs for SMEFT as well as WET are known at the 1-loop level [13-17]. The recent results for ADMs in the SMEFT extended with right-handed neutrino fields can be found in refs. [18-20]. Based on these calculations, the tools such as wilson [21] and DsixTools [22, 23] have been developed. (See also ref. [24] for a discussion on the analytic solutions to the SMEFT RGEs). Using these codes, it is possible to include the RG running effects in the theoretical predictions which can be obtained using flavio [25]. Several other packages [26-28] exist to facilitate the different kinds of tasks for studying the phenomenology in the SMEFT framework. As far as the present work is concerned, we have used flavio for the theoretical predictions and the RG running effects have been taken care by using the wilson package.

In this work, we focus on a subset of SMEFT operators which contain both lepton and quark fields currents:

$$
\left(\bar{L}_{i} \gamma_{\mu} L_{j}\right)\left(\bar{Q}_{k} \gamma^{\mu} Q_{l}\right)
$$

here, $i, j, k, l$ denote the flavour indices. Such operators are known as the semileptonic operators. It is well-known that, at tree-level, these operators enter into various semileptonic decays of mesons. In this context, the operators which violate the quark flavour have been extensively studied in the literature [8, 9, 29-36]. On the other hand, a generic flavour structure of these operators is not yet fully explored. In particular, the quark flavour conserving counterparts deserve more attention. A given NP model can generate both flavour violating as well as conserving operators, therefore, it is essential to know what kind of constraints apply on the later ones. One of the goals of the present work is to fill this gap by identifying all possible low energy and EW scale observables, which can be used to probe a generic flavour structure of the semileptonic operators. Concerning this matter, the ref. [37] discusses the contribution of semileptonic operators to the EWP observables, assuming flavour universality. Similarly, the refs. [7, 38], pointed out the importance of EWP and lepton flavour violating (LFV) constraints on the semileptonic operators needed to resolve the $B$-anomalies. For more recent studies on this topic, see also refs. [30, 32, 39]. Due to the reasons outlined above, we will restrict ourself to the flavour structures such that there is no quark-flavour violation, to begin with, at the scale $\Lambda$. In other words, the operators in which are interested are either flavour conserving in both currents or at most they violate the lepton flavour at the NP scale. We will emphasize the importance RG running from $\Lambda$ to the EW scale, through which these operators can contribute to a 
verity of observables at lower scales. In this regard, we will first isolate the most important terms in the ADMs due to gauge couplings as well as Yukawas, i.e., the ones which are phenomenologically relevant, given the current precision of the measurements.

The remainder of the paper is structured as follows. In section 2, we will discuss our strategy, and in section 3, we discuss the SMEFT RG running of semileptonic operators. In section 4 , we will identify various observables which are relevant for the different flavour structures of the operators under consideration. In section 5, we will discuss the sensitivities to the NP scales $\Lambda$ for various operators. Finally, we move on to the conclusions in section 6 . Additional material is collected in appendices A, B, and C.

\section{General strategy}

In SMEFT, the SM is extended with a series of higher dimensional effective operators invariant under the full gauge symmetry of the SM. In general, the SMEFT Lagrangian can be written as

$$
\mathcal{L}_{\mathrm{SMEFT}}^{d \geq 5}=\sum_{\mathcal{O}_{a}^{\dagger}=\mathcal{O}_{a}} \mathcal{C}_{a} \mathcal{O}_{a}+\sum_{\mathcal{O}_{a}^{\dagger} \neq \mathcal{O}_{a}}\left(\mathcal{C}_{a} \mathcal{O}_{a}+\mathcal{C}_{a}^{*} \mathcal{O}_{a}^{\dagger}\right)
$$

here, $\mathcal{C}_{a}$ are known to be the Wilson coefficients. A complete list of SMEFT operators can be found in refs. [1, 2]. In this work, we will focus on a subset, the four-fermion semileptonic operators:

$$
\begin{aligned}
{\left[\mathcal{O}_{\ell q}^{(1)}\right]_{i j k l} } & =\left(\bar{\ell}_{i} \gamma_{\mu} \ell_{j}\right)\left(\bar{q}_{k} \gamma^{\mu} q_{l}\right), \\
{\left[\mathcal{O}_{\ell q}^{(3)}\right]_{i j k l} } & =\left(\bar{\ell}_{i} \gamma_{\mu} \tau^{a} \ell_{j}\right)\left(\bar{q}_{k} \gamma^{\mu} \tau^{a} q_{l}\right), \\
{\left[\mathcal{O}_{\ell u}\right]_{i j k l} } & =\left(\bar{\ell}_{i} \gamma_{\mu} \ell_{j}\right)\left(\bar{u}_{k} \gamma^{\mu} u_{l}\right), \\
{\left[\mathcal{O}_{\ell d}\right]_{i j k l} } & =\left(\bar{\ell}_{i} \gamma_{\mu} \ell_{j}\right)\left(\bar{d}_{k} \gamma^{\mu} d_{l}\right), \\
{\left[\mathcal{O}_{e d}\right]_{i j k l} } & =\left(\bar{e}_{i} \gamma_{\mu} e_{j}\right)\left(\bar{d}_{k} \gamma^{\mu} d_{l}\right), \\
{\left[\mathcal{O}_{e u}\right]_{i j k l} } & =\left(\bar{e}_{i} \gamma_{\mu} e_{j}\right)\left(\bar{u}_{k} \gamma^{\mu} u_{l}\right), \\
{\left[\mathcal{O}_{q e}\right]_{i j k l} } & =\left(\bar{q}_{i} \gamma_{\mu} q_{j}\right)\left(\bar{e}_{k} \gamma^{\mu} e_{l}\right),
\end{aligned}
$$

here, the flavour indices $i, j, k, l$ can take values from 1 to 3 and $q, u, d, \ell, e$ represent the quark doublet, right-handed up-type quark, right-handed down-type quark, lepton doublet and right-handed lepton fields, respectively. The down-type quarks are chosen to be in the mass basis at the scale $\Lambda$. This corresponds to the Warsaw-down basis of the SMEFT [40]. For convenience, we divide the operators into three classes based on their flavour structure at the scale $\Lambda$ :

- $\Delta F=(0,0)$ : the operators which do not violate quark ${ }^{1}$ and lepton flavours,

\footnotetext{
${ }^{1}$ Since we work in the Warsaw-down basis, the Cabibbo-Kobayashi-Maskawa (CKM) rotations can give rise to quark flavour violating operators in the up-sector, even at the scale $\Lambda$. Note that the CKM matrix itself can be affected by NP [39, 41], however such effects are beyond the scope of present analyses. In our analyses for numerics the CKM is obtained with wilson program using the input $V_{u s}=0.2243, V_{u b}=$ $3.62 \times 10^{-3}, V_{c b}=4.221 \times 10^{-2}, \delta=1.27$ at the $Z$-mass scale.
} 
- $\Delta F=(1,0)$ : the operators which do violate lepton flavour by one unit but do not violate the quark flavour,

- $\Delta F=(i, 1)$ : the operators which do violate quark flavour by one unit but may or may not violate the lepton flavour, i.e, $i=0$ or 1 .

Since, the $\Delta F=(i, 1)$ type operators are already well studied in the literature, we will not consider them here. On integrating out the new degrees of freedom at $\Lambda$, a unique tower of SMEFT operators is generated. However, a priori it is not obvious to which observables these operators can enter into at the lower energies. This is because of three reasons. Firstly, the $\mathrm{SU}(2)_{L}$ invariance can lead to correlations between different type of observables. Secondly, the pattern of mixing between different operators due to running from $\Lambda$ to the EW scale is very complex in nature. Often this leads to the appearance of new operators at the EW scale, which can give rise to unpredictable correlations among low-energy observables. Finally, the choice of the flavour basis at the NP scale is not invariant with respect to the RG evolution. Therefore, it is extremely important to systematically analyze these effects for all flavour structures of the operators of our interest. This will allow us to identify all possible observables which are sensitive to these operators.

With these motivations, first we will identify the most sensitive observables which can be used to probe the operators listed in $(2.2)-(2.8)$ for the following flavour combinations:

$$
\begin{aligned}
& \Delta F=(0,0): 1111,1122,1133,2211,2222,2233,3311,3322,3333, \\
& \Delta F=(1,0): 1211,1222,1233,1311,1322,1333,2311,2322,2333 .
\end{aligned}
$$

Note that none of these flavour combinations are quark flavour violating. Next, we will proceed in three steps: to begin with, we will study the operator mixing pattern due RG evolution for the two classes of the flavour structures as shown in eqs. (2.9)-(2.10). Based on this, we will then identify all possible observables which can be used to constrain a given operator directly (at tree-level) or through the operators to which it mixes into, through the RG effects (at 1-loop level). Using this information, we will finally derive the lower bounds on the scale of each semileptonic operator assuming the presence of a single operator at the scale $\Lambda$.

\section{Renormalization group running}

In this section we discuss the RG running of the SMEFT semileptonic Wilson coefficients. In general, the running is governed by the coupled differential equations

$$
\dot{\mathcal{C}}(\mu) \equiv 16 \pi^{2} \frac{d \mathcal{C}(\mu)}{d \ln \mu}=\hat{\gamma}(\mu) \mathcal{C}(\mu)
$$

with

$$
\mathcal{C}(\mu)=\left(\mathcal{C}_{1}(\mu), \mathcal{C}_{2}(\mu), \ldots\right)^{T}
$$

Here, $\mu$ is the renormalization scale and $\hat{\gamma}$ is the anomalous dimension matrix which is function of the SM parameters such as gauge and Yukawa couplings. In the leading-log (LL) 
approximation, the solution to these equations for running from scale $\Lambda$ and $\mu$ reads

$$
\mathcal{C}(\mu)=\left[\hat{1}-\frac{\hat{\gamma}}{16 \pi^{2}} \ln \left(\frac{\Lambda}{\mu}\right)\right] \mathcal{C}(\Lambda) .
$$

In the following we analyze the RG running and operator mixing of various quark-flavour conserving semileptonic operators (shown in eqs. (2.2)-(2.8)) of our interest to various other operators. These effects can potentially relate them to new observables generated at 1-loop level and hence allow us to put additional constraints. Using the ADMs calculated in refs. [13-16] we find that, depending upon the flavour structures, the quark flavour conserving semileptonic operators can mix with two types of operators. The first category is the $\psi^{2} \phi^{2} D$ type operators:

$$
\begin{aligned}
{\left[\mathcal{O}_{\phi \ell}^{(1)}\right]_{i j} } & =\left(\phi^{\dagger} i \overleftrightarrow{D_{\mu}} \phi\right)\left(\bar{\ell}_{i} \gamma^{\mu} \ell_{j}\right), \\
{\left[\mathcal{O}_{\phi \ell}^{(3)}\right]_{i j} } & =\left(\phi^{\dagger} i \overleftrightarrow{D_{\mu}^{I}} \phi\right)\left(\bar{\ell}_{i} \tau^{I} \gamma^{\mu} \ell_{j}\right) \\
{\left[\mathcal{O}_{\phi e}\right]_{i j} } & =\left(\phi^{\dagger} i \overleftrightarrow{D_{\mu}} \phi\right)\left(\bar{e}_{i} \gamma^{\mu} e_{j}\right), \\
{\left[\mathcal{O}_{\phi q}^{(1)}\right]_{i j} } & =\left(\phi^{\dagger} i \overleftrightarrow{D_{\mu}} \phi\right)\left(\bar{q}_{i} \gamma^{\mu} q_{j}\right), \\
{\left[\mathcal{O}_{\phi q}^{(3)}\right]_{i j} } & =\left(\phi^{\dagger} i \overleftrightarrow{D_{\mu}^{I}} \phi\right)\left(\bar{q}_{i} \tau^{I} \gamma^{\mu} q_{j}\right) \\
{\left[\mathcal{O}_{\phi u}\right]_{i j} } & =\left(\phi^{\dagger} i \overleftrightarrow{D_{\mu}} \phi\right)\left(\bar{u}_{i} \gamma^{\mu} u_{j}\right), \\
{\left[\mathcal{O}_{\phi d}\right]_{i j} } & =\left(\phi^{\dagger} i \overleftrightarrow{D_{\mu}} \phi\right)\left(\bar{d}_{i} \gamma^{\mu} d_{j}\right)
\end{aligned}
$$

Here, $I$ is the $\mathrm{SU}(2)$ index and $D_{\mu}$ stands for the covariant derivative. Secondly, they also mix with the purely leptonic operators given by

$$
\begin{aligned}
& {\left[\mathcal{O}_{\ell \ell}\right]_{i j k l}=\left(\bar{\ell}_{i} \gamma_{\mu} \ell_{j}\right)\left(\bar{\ell}_{k} \gamma^{\mu} \ell_{l}\right),} \\
& {\left[\mathcal{O}_{\ell e}\right]_{i j k l}=\left(\bar{\ell}_{i} \gamma_{\mu} \ell_{j}\right)\left(\bar{e}_{k} \gamma^{\mu} e_{l}\right),} \\
& {\left[\mathcal{O}_{e e}\right]_{i j k l}=\left(\bar{e}_{i} \gamma_{\mu} e_{j}\right)\left(\bar{e}_{k} \gamma^{\mu} e_{l}\right) .}
\end{aligned}
$$

In addition, the $\Delta F=(0,0)$ type semileptonic operators can also mix with the fourquark operators, however we do not find any significant constraints due to such an operator mixing. In order to get the general picture, for our qualitative discussion, we will use the LL solutions to the corresponding RG equations as given by eq. (3.3). However, for the numerics, we sum the logs using full numerical solutions which have been obtained using the wilson program [21].

\subsection{Evolution of $\Delta F=(0,0)$ operators}

The ADMs in the SMEFT depend on the gauge couplings as well the Yukawas. In this section, we identify the phenomenologically important terms in the ADMs of $\Delta F=(0,0)$ semileptonic operators.

\subsubsection{Operator mixing due to gauge interactions}

First, we discuss the operator mixing of the $\Delta F=(0,0)$ semileptonic operators due the gauge couplings. The flavour combinations for these operators are specified in eq. (2.9). 
Therefore, as an initial condition, at NP scale, only the Wilson coefficients of the operators given in (2.2)-(2.8) are assumed to be non-zero. It turns out that, these operators can mix with the $\psi^{2} \phi^{2} D$-type operators, which are listed in eqs. (3.4)-(3.10), through EW interactions. Now we use the LL approximation to relate the Wilson coefficients of the semileptonic operators at the scale $\Lambda$ to the Wilson coefficients of $\psi^{2} \phi^{2} D$ operators which get generated at the EW scale due to the operator mixing. We find

$$
\left(\begin{array}{l}
{\left[\mathcal{C}_{\phi \ell}^{(1)}\right]_{i i}} \\
{\left[\mathcal{C}_{\phi \ell}^{(3)}\right]_{i i}} \\
{\left[\mathcal{C}_{\phi e}\right]_{i i}} \\
{\left[\mathcal{C}_{\phi q}^{(1)}\right]_{k k}} \\
{\left[\mathcal{C}_{\phi q}^{(3)}\right]_{k k}} \\
{\left[\mathcal{C}_{\phi d}\right]_{k k}} \\
{\left[\mathcal{C}_{\phi u}\right]_{k k}}
\end{array}\right)=L\left(\begin{array}{ccccccc}
\frac{2}{3} g_{1}{ }^{2} & 0 & \frac{4}{3} g_{1}{ }^{2} & -\frac{2}{3} g_{1}{ }^{2} & 0 & 0 & 0 \\
0 & 2 g_{2}^{2} & 0 & & 0 & 0 & 0 \\
0 & 0 & 0 & 0 & -\frac{2}{3} g_{1}{ }^{2} & \frac{4}{3} g_{1}{ }^{2} & \frac{2}{3} g_{1}{ }^{2} \\
-\frac{2}{3} g_{1}{ }^{2} & 0 & 0 & 0 & 0 & 0 & -\frac{2}{3} g_{1}{ }^{2} \\
0 & \frac{2}{3} g_{2}^{2} & 0 & 0 & 0 & 0 & 0 \\
0 & 0 & 0 & -\frac{2}{3} g_{1}{ }^{2} & -\frac{2}{3} g_{1}{ }^{2} & 0 & 0 \\
0 & 0 & -\frac{2}{3} g_{1}{ }^{2} & 0 & 0 & -\frac{2}{3} g_{1}{ }^{2} & 0
\end{array}\right)\left(\begin{array}{c}
{\left[\mathcal{C}_{\ell q}^{(1)}\right]_{i i k k}} \\
{\left[\mathcal{C}_{\ell q}^{(3)}\right]_{i i k k}} \\
{\left[\mathcal{C}_{\ell u}\right]_{i i k k}} \\
{\left[\mathcal{C}_{\ell d}\right]_{i i k k}} \\
{\left[\mathcal{C}_{e d}\right]_{i i k k}} \\
{\left[\mathcal{C}_{e u}\right]_{i i k k}} \\
{\left[\mathcal{C}_{q e}\right]_{k k i i}}
\end{array}\right)
$$

Strictly speaking on the l.h.s. we should have written the difference between the Wilson coefficients at the EW scale and their corresponding values at the scale $\Lambda$, i.e.,

$$
\delta \mathcal{C}\left(\mu_{\mathrm{ew}}\right)=\mathcal{C}\left(\mu_{\mathrm{ew}}\right)-\mathcal{C}(\Lambda)
$$

However, since at the scale $\Lambda$ we assume only the semileptonic operators to be non-zero, we have $\mathcal{C}(\Lambda)=0$ and therefore $\delta \mathcal{C}\left(\mu_{\text {ew }}\right)=\mathcal{C}\left(\mu_{\text {ew }}\right)$. Also, we have expressed the loop suppression factor and the $\log$ in terms of the quantity $L$, given by

$$
L=\frac{1}{16 \pi^{2}} \ln \left(\frac{\mu_{\mathrm{ew}}}{\Lambda}\right)
$$

In eq. (3.14), the $g_{1}$ and $g_{2}$ are the EW gauge couplings at the scale $\Lambda$. It is important to note that for the first three (last four) elements on the l.h.s., only the repeated index $k(i)$ is summed over on the r.h.s. However, on the 1.h.s. indices $k$ and $i$ are not summed over and can take values in the range 1-3. For simplicity, we do not show the self-mixing of the operators. Interestingly, after the EW symmetry breaking, the $\psi^{2} \phi^{2} D$ operators are known to give corrections to the $W$ and $Z$ boson couplings with quarks and leptons [42-45] (see also more recent refs. [37, 39, 46-51]). Therefore, the quark-flavour conserving semileptonic operators can be indirectly probed through EWP data.

In this context, one should also consider the operator $\left[\mathcal{C}_{\ell \ell}\right]_{1221}$ which enters into the muon decay, i.e., $\mu \rightarrow e \nu \bar{\nu}$ and hence affects the extraction of Fermi constant $G_{F}$. At the EW scale the Wilson coefficient $\left[\mathcal{C}_{\ell \ell}\right]_{1221}$ can be written as

$$
\left[\mathcal{C}_{\ell \ell}\right]_{1221}=4 g_{2}^{2} L\left[\mathcal{C}_{\ell q}^{(3)}\right]_{i i k k},
$$

here the indices on the r.h.s. are summed over $i i=11,22$ and $k k=11,22,33$.

In addition, we find the mixing between $\left[\mathcal{C}_{\ell q}^{(1)}\right]$ and $\left[\mathcal{C}_{\ell q}^{(3)}\right]$ to be phenomenologically relevant. At the 1-loop level, this goes through the EW corrections. Therefore, the strength 
of mixing is driven by the gauge coupling $g_{2}$. As before, solving the corresponding RGEs in LL approximation, one finds

$$
\left(\begin{array}{l}
{\left[\mathcal{C}_{\ell q}^{(1)}\right]_{i i k k}} \\
{\left[\mathcal{C}_{\ell q}^{(3)}\right]_{i i k k}}
\end{array}\right)=L\left(\begin{array}{cc}
- & 9 g_{2}^{2} \\
3 g_{2}^{2} & -
\end{array}\right)\left(\begin{array}{l}
{\left[\mathcal{C}_{\ell q}^{(1)}\right]_{i i k k}} \\
{\left[\mathcal{C}_{\ell q}^{(3)}\right]_{i i k k}}
\end{array}\right),
$$

here, the repeated indices $k$ and $i$ on the l.h.s. as well as on the r.h.s. are not summed over. Once again, we have suppressed the self-mixing (indicated by the entries with dashes) of these operators and $\log$ term $L$ is given by (3.16). The mixing of $\left[\mathcal{C}_{\ell q}^{(1)}\right]_{i i k k}$ with $\left[\mathcal{C}_{\ell q}^{(3)}\right]_{i i k k}$ can induce new charged current transitions after EW symmetry breaking.

\subsubsection{Operator mixing due to top-Yukawa interactions}

In this subsection, we isolate phenomenologically important terms in the ADMs which depend on the Yukawa interactions. In this regard, we will keep only the largest terms involving the top-Yukawa coupling. Solving the RGEs in the LL approximation, we find

$$
\left(\begin{array}{l}
{\left[\mathcal{C}_{\phi \ell}^{(1)}\right]_{i j}} \\
{\left[\mathcal{C}_{\phi \ell}^{(3)}\right]_{i j}} \\
{\left[\mathcal{C}_{\phi e}\right]_{i j}}
\end{array}\right) \simeq 6 y_{t}^{2} L\left(\begin{array}{ccccc}
1 & 0 & -1 & 0 & 0 \\
0 & -1 & 0 & 0 & 0 \\
0 & 0 & 0 & 1 & -1
\end{array}\right)\left(\begin{array}{l}
{\left[\mathcal{C}_{\ell q}^{(1)}\right]_{i j 33}} \\
{\left[\mathcal{C}_{\ell q}^{(3)}\right]_{i j 33}} \\
{\left[\mathcal{C}_{\ell u}\right]_{i j 33}} \\
{\left[\mathcal{C}_{q e}\right]_{33 i j}} \\
{\left[\mathcal{C}_{e u}\right]_{i j 33}}
\end{array}\right)
$$

here, $y_{t}$ is the Yukawa coupling for the top-quark. In eq. (3.19), for $\Delta F=(0,0)$ operators the flavour indices $i$ and $j$ can be set equal. It is worth mentioning that for simplicity, we have not included the non-standard effects due to running of the Yukawa couplings themselves, which can lead to additional contributions to the quark-flavour violating semileptonic operators at the EW scale. We will return to this point in section 4.1.2.

\subsection{Evolution of $\Delta F=(1,0)$ operators}

Now we consider the $\Delta F=(1,0)$ type semileptonic operators which violate the leptonic flavour by one unit but conserve the quark flavour (see eq. (2.10)). Apart from the selfmixing, these operators mix with the lepton flavour violating leptonic and $\psi^{2} \phi^{2} D$ type operators, $\left[\mathcal{C}_{\phi \ell}^{(1)}\right]_{i j},\left[\mathcal{C}_{\phi \ell}^{(1)}\right]_{i j}$ and $\left[\mathcal{C}_{\phi e}\right]_{i j}$ with $i \neq j$, through the EW interactions. Once again, solving the RG equations in the LL approximation, we find,

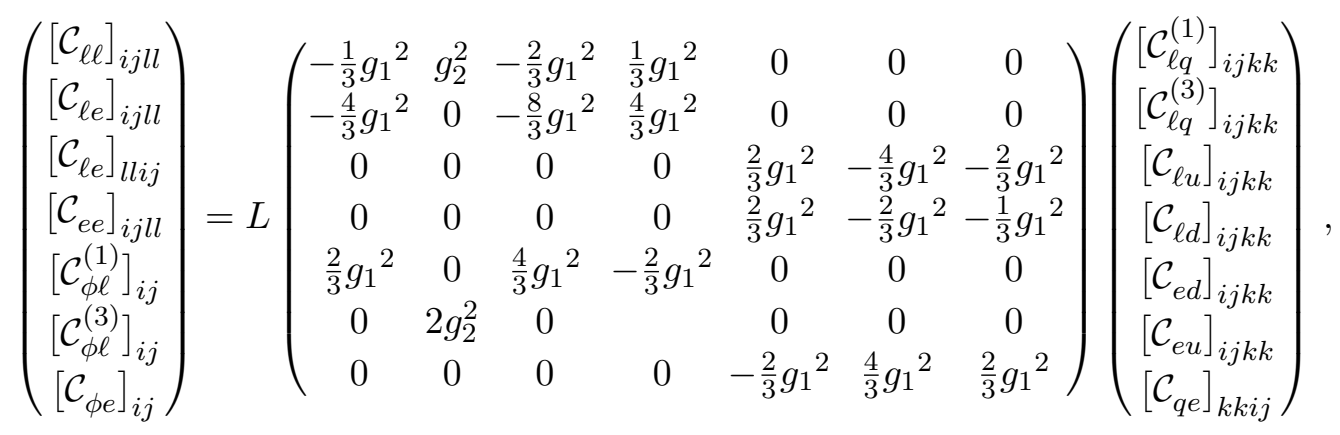


here the repeated indices $k$ on the r.h.s. is summed over the values $1-3$, and $i \neq j$ holds on the both sides. Also, the repeated index $l$ on the l.h.s. can take values in the range $1-3$ but it is not summed over. The logarithmic piece $L$ is defined in equation (3.16). The operators on the l.h.s. contribute to the LFV $W, Z$ boson couplings and leptonic decays. We will come to this point later with more details. Note that, below the EW scale, the semileptonic operators can mix into purely leptonic operators also through QED interaction [52, 53].

\section{Observables induced at 1-loop level}

Based on our discussions in the previous section, we are now in position to identify the 1-loop induced observables to which the semileptonic operators can contribute. We will see how the SMEFT RG running can play an important role in this respect. As discussed before, because of the complicated operator mixing pattern in SMEFT, the semileptonic operators can contribute to observables of very different nature depending upon the operators with which they mix, which can be read out from the l.h.s. of eqs. (3.14), (3.17), (3.18), (3.19) and (3.20). In order to get a general picture, we will present the expressions for the relevant low energy dim- 4 and dim- 6 couplings in terms of semileptonic Wilson coefficients at the high scale by employing the LL approximation. Eventually, we will sum the logs with the help of numerical solutions.

\subsection{Observables for $\Delta F=(0,0)$ operators}

We have identified three categories of the observables which are relevant for $\Delta F=(0,0)$ type operators. Those are the EWP observables, flavour violating $B$-decays and charged current decays. In the following, we discuss how various semileptonic operators can contribute to them through operator mixing.

\subsubsection{Electroweak precision observables}

As indicated by eq. (3.14), the $\Delta F=(0,0)$ semileptonic operators can mix with the $\psi^{2} \phi^{2} D$ type operators due to electroweak interactions. In addition, this mixing also depends on the top-Yukawa interactions, see e.g. eq. (3.19). Interestingly, after EW symmetry breaking the latter operators give corrections to the $Z$ and $W$ boson couplings with the fermions. Using the LL solutions to the RGEs, as presented before, we can express the NP contributions to these couplings directly in terms of the Wilson coefficients of semileptonic operators at the high scale $\Lambda$. In general, NP shifts in the neutral $Z$ boson couplings with the fermions can be parameterized as

$$
\mathcal{L}_{Z} \supset g_{Z} \sum_{\psi=u, d, e, \nu_{L}} \bar{\psi}_{i} \gamma^{\mu}\left[\delta\left(g_{L}^{\psi}\right)_{i j} P_{L}+\delta\left(g_{R}^{\psi}\right)_{i j} P_{R}\right] \psi_{j} Z_{\mu}
$$

here $g_{Z}=-g_{2} / \cos \theta_{W}$ and $\theta_{W}$ represents the weak-mixing angle. NP can enter into $\delta g_{X}^{\psi}$ with $X=L, R$, through three difference sources. This can be understood from the equation [49]

$$
\delta\left(g_{X}^{\psi}\right)_{i j}=\delta g_{Z}\left(g_{X}^{\psi, \mathrm{SM}}\right)_{i j}-Q_{\psi} \delta \sin ^{2} \theta_{W} \delta_{i j}+\delta\left(g_{X}^{\psi}\right)_{i j}^{\mathrm{dir}}
$$


here, the contributions in the first two and the last term can be thought of as indirect and direct shifts to the $Z$ boson couplings, respectively. In SMEFT, the tree-level expressions for the quantities $\delta g_{Z}, \delta \sin ^{2} \theta_{W}$ and $\delta\left(g_{X}^{\psi}\right)_{i j}$ can be found in appendix A. Also, $Q_{\psi}$ represents the electric charge and $g_{L}^{\psi, \mathrm{SM}}=T_{3}-Q_{\psi} \sin ^{2} \theta_{W}, g_{R}^{\psi, \mathrm{SM}}=-Q_{\psi} \sin ^{2} \theta_{W}$. Below we present the 1-loop contributions to all these quantities due to semileptonic operators in the LL approximation. Using the eqs. (3.14) and (A.1)-(A.7) one can obtain the NP shifts in the $Z$ couplings with quarks:

$$
\begin{aligned}
& \delta\left(g_{L}^{u}\right)_{k k}^{\mathrm{dir}}=-\frac{g_{1}^{2}}{3} v^{2} L V_{k m}\left(-\left[\mathcal{C}_{\ell q}^{(1)}\right]_{i i m n}-\frac{g_{2}^{2}}{g_{1}^{2}}\left[\mathcal{C}_{\ell q}^{(3)}\right]_{i i m n}-\left[\mathcal{C}_{q e}\right]_{m n i i}\right) V_{n k}^{\dagger}, \\
& \delta\left(g_{R}^{u}\right)_{k k}^{\mathrm{dir}}=-\frac{g_{1}^{2}}{3} v^{2} L\left(-\left[\mathcal{C}_{\ell u}^{(1)}\right]_{i i k k}-\left[\mathcal{C}_{e u}^{(3)}\right]_{i i k k}\right), \\
& \delta\left(g_{L}^{d}\right)_{k k}^{\mathrm{dir}}=-\frac{g_{1}^{2}}{3} v^{2} L\left(-\left[\mathcal{C}_{\ell q}^{(1)}\right]_{i i k k}+\frac{g_{2}^{2}}{g_{1}^{2}}\left[\mathcal{C}_{\ell q}^{(3)}\right]_{i i k k}-\left[\mathcal{C}_{q e}\right]_{k k i i}\right), \\
& \delta\left(g_{R}^{d}\right)_{k k}^{\mathrm{dir}}=-\frac{g_{1}^{2}}{3} v^{2} L\left(-\left[\mathcal{C}_{\ell d}\right]_{i i k k}-\left[\mathcal{C}_{e d}\right]_{i i k k}\right) .
\end{aligned}
$$

Similarly, the shifts in the $Z$ boson couplings with leptons are found to be

$$
\begin{aligned}
\delta\left(g_{L}^{\nu}\right)_{i i}^{\text {dir }}= & -\frac{g_{1}^{2}}{3} v^{2} L\left(\left[\mathcal{C}_{\ell q}^{(1)}\right]_{i i k k}-\frac{3 g_{2}^{2}}{g_{1}^{2}}\left[\mathcal{C}_{\ell q}^{(3)}\right]_{i i k k}+2\left[\mathcal{C}_{\ell u}\right]_{i i k k}-\left[\mathcal{C}_{\ell d}\right]_{i i k k}\right) \\
& -6 v^{2} y_{t}^{2} L\left(\left[\mathcal{C}_{\ell q}^{(1)}\right]_{i i 33}+\left[\mathcal{C}_{\ell q}^{(3)}\right]_{i i 33}-\left[\mathcal{C}_{\ell u}\right]_{i i 33}\right), \\
\delta\left(g_{L}^{e}\right)_{i i}^{\text {dir }}= & -\frac{g_{1}^{2}}{3} v^{2} L\left(\left[\mathcal{C}_{\ell q}^{(1)}\right]_{i i k k}+\frac{3 g_{2}^{2}}{g_{1}^{2}}\left[\mathcal{C}_{\ell q}^{(3)}\right]_{i i k k}+2\left[\mathcal{C}_{\ell u}\right]_{i i k k}-\left[\mathcal{C}_{\ell d}\right]_{i i k k}\right) \\
& -6 v^{2} y_{t}^{2} L\left(\left[\mathcal{C}_{\ell q}^{(1)}\right]_{i i 33}-\left[\mathcal{C}_{\ell q}^{(3)}\right]_{i i 33}-\left[\mathcal{C}_{\ell u}\right]_{i i 33}\right), \\
\delta\left(g_{R}^{e}\right)_{i i}^{\text {dir }}= & -\frac{g_{1}^{2}}{3} v^{2} L\left(-\left[\mathcal{C}_{e d}\right]_{i i k k}+2\left[\mathcal{C}_{e u}\right]_{i i k k}+\left[\mathcal{C}_{q e}\right]_{k k i i}\right) \\
& -6 v^{2} y_{t}^{2} L\left(\left[\mathcal{C}_{q e}\right]_{33 i i}-\left[\mathcal{C}_{e u}\right]_{i i 33}\right) .
\end{aligned}
$$

Here, $v=246 \mathrm{GeV}$ is the vacuum expectation value and $L$ is given by (3.16). Similarly, for $\delta g_{Z}$ and $\delta \sin ^{2} \theta_{W}$ are found to be

$$
\begin{aligned}
\delta g_{Z} & =3 v^{2} y_{t}^{2} L\left(\left[\mathcal{C}_{\ell q}^{(3)}\right]_{1133}+\left[\mathcal{C}_{\ell q}^{(3)}\right]_{2233}\right) \\
\delta \sin ^{2} \theta_{W} & =-\frac{\sin ^{2} 2 \theta_{W}}{4 \cos 2 \theta_{W}} 6 v^{2} y_{t}^{2} L\left(\left[\mathcal{C}_{\ell q}^{(3)}\right]_{1133}+\left[\mathcal{C}_{\ell q}^{(3)}\right]_{2233}\right) .
\end{aligned}
$$

On the basis of the above discussions, now we point out a few important observations:

- First, note that the RG induced EW corrections to $\delta g_{Z}$ and $\delta \sin ^{2} \theta_{W}$ due to $\left[\mathcal{C}_{\ell q}^{(3)}\right]_{i i k k}$ for $i i=11,22$ and $k k=11,22,33$, get canceled among $\left[\mathcal{C}_{\phi \ell}^{(3)}\right]_{11},\left[\mathcal{C}_{\phi \ell}^{(3)}\right]_{22}$ and $\left[\mathcal{C}_{\ell \ell}\right]_{1221}$. This can be seen by inserting the LL contributions of $\left[\mathcal{C}_{\ell q}^{(3)}\right]_{i i k k}$ from eqs. (3.17) and (3.14) to the latter Wilson coefficients in eqs. (A.8)-(A.9). However, this is no longer true once the contribution due to the Yukawa couplings, as shown in eq. (3.19), is included. 
- Next, in general, the top-Yukawa dependent contributions, given in eqs. (4.10)-(4.11), are larger in size as compared to the direct EW corrections to the $Z$-couplings shown in eqs. (4.3)-(4.9). So, for the cases in which both effects exist simultaneously, the former has a greater impact.

- Furthermore, it is evident from eq. (4.2) that the impact of the $\left[\mathcal{C}_{\ell q}^{(3)}\right]_{i i 33}$ for $i i=11$ or 22 on $\delta\left(g_{X}^{Y}\right)_{i i}$ through $\delta g_{Z}$ and $\delta \sin ^{2} \theta_{W}$ is universal for all three families of leptons and quarks. On the other hand the shifts $\delta\left(g_{L}^{e}\right)_{i i}^{\text {dir }}, \delta\left(g_{R}^{e}\right)_{i i}^{\text {dir }}$ and $\delta\left(g_{L}^{\nu}\right)_{i i}^{\text {dir }}$ also experience effects due to the top-Yukawa interactions, which however are lepton flavour dependent.

- Finally, the contributions of the semileptonic operators due to top-Yukawa effects do not affect the $Z$ boson couplings to quarks directly. This is however still possible through $\delta g_{Z}$ and $\delta \sin ^{2} \theta_{W}$, but only for the case of $\left[\mathcal{C}_{\ell q}^{(3)}\right]_{i i 33}$ with $i i=11,22$.

Next, we look at the impact of semileptonic operators on the $W$-boson couplings which can be parameterized as

$$
\mathcal{L}_{W} \supset \frac{-e}{\sqrt{2} \sin \theta_{W}}\left(\delta\left(\varepsilon_{L}^{\ell}\right)_{i j}^{\mathrm{dir}} \bar{\nu}_{i} \gamma_{\mu} P_{L} e_{j}+\delta\left(\varepsilon_{L}^{q}\right)_{i j}^{\mathrm{dir}} \bar{u}_{i} \gamma_{\mu} P_{L} d_{j}\right) W_{+}^{\mu}+\text { h.c. }
$$

here the NP shifts are given as [49]

$$
\delta\left(\varepsilon_{L}^{\psi}\right) i i=\delta\left(\varepsilon_{L}^{\psi}\right)_{i i}^{\operatorname{dir}}-\frac{1}{2} \frac{\delta \sin ^{2} \theta_{W}}{\sin ^{2} \theta_{W}} .
$$

Using the LL solutions, we find the shifts $\delta\left(\varepsilon_{L}^{\psi}\right)_{i i}^{\text {dir }}$ in terms of the semileptonic operators to be

$$
\begin{aligned}
\delta\left(\varepsilon_{L}^{\ell}\right)_{i i}^{\operatorname{dir}} & =2 g_{2}^{2} L\left[\mathcal{C}_{\ell q}^{(3)}\right]_{i i k k}-6 v^{2} y_{t}^{2} L\left[\mathcal{C}_{\ell q}^{(3)}\right]_{i i 33}, \\
\delta\left(\varepsilon_{L}^{q}\right)_{k k}^{\text {dir }} & =\frac{2}{3} g_{2}^{2} L V_{k m}\left[\mathcal{C}_{\ell q}^{(3)}\right]_{i i m k} .
\end{aligned}
$$

We make the following observations for the RG induced shifts in the $W$ couplings:

- The $W$ couplings to both quarks and leptons are universally affected for all three families by $\left[\mathcal{C}_{\ell q}^{(3)}\right]_{i i 33}$ for $i i=11,22$ through $\delta \sin ^{2} \theta_{W}$.

- The top-Yukawa effects do not give direct contributions to the $W$ couplings.

- The leptonic couplings can be directly affected by $\left[\mathcal{C}_{\ell q}^{(3)}\right]_{i i 33}$ for $i i=11,22,33$ through top-Yukawa interactions. This effect is however flavour dependent.

In order to quantify these effects, in appendix B we report tables for the numerical values of the RG induced shifts in the $Z$ and $W$ couplings at the EW scale due to semileptonic operators present at the NP scale $\Lambda$. To understand the relative importance of the top-Yukawa and gauge interactions, we present two sets of numbers with and without Yukawa RG running effects. It is evident that, whenever present, the top-Yukawa effects always dominate. 
Now, given that the $Z$ and $W$ boson couplings are strongly constrained by the EWP observables, this implies that the flavour conserving semileptonic $\Delta F=(0,0)$ type operators can be indirectly constrained by the EWP measurements. The following list of operators can be constrained through this mechanism:

$$
\left[\mathcal{C}_{\ell q}^{(1)}\right]_{i j k l},\left[\mathcal{C}_{\ell q}^{(3)}\right]_{i j k l},\left[\mathcal{C}_{e d}\right]_{i j k l},\left[\mathcal{C}_{e u}\right]_{i j k l},\left[\mathcal{C}_{\ell u}\right]_{i j k l},\left[\mathcal{C}_{\ell d}\right]_{i j k l},\left[\mathcal{C}_{q e}\right]_{i j k l},
$$

with

$$
i j k l \equiv 1111,1122,1133,2211,2222,2233,3311,3322,3333 .
$$

In the next section, we will use the EWP measurements as constraints to derive the lower bounds on the NP scales of the flavour conserving semileptonic operators in given eq. (4.16). To this end, the list of EWP observables [54-60] used in our analysis are taken from table 13 of ref. [39]. In addition, we have also included a recent measurement of the ratio $R_{\tau \mu}(W \rightarrow \ell \bar{\nu})=\mathcal{B}(W \rightarrow \tau \bar{\nu}) / \mathcal{B}(W \rightarrow \mu \bar{\nu})[61]$.

\subsection{2 $B$ meson decays}

Throughout the analyses, we have used Warsaw-down basis at the high scale. In this basis, to begin with the down-type quark and lepton mass matrices are diagonal, whereas, the up-type quark matrix takes the form $V^{\dagger} \operatorname{diag}\left(y_{u}, y_{c}, y_{t}\right)$. Here, $V$ represents the CKM matrix. However, due to the RG running of the Yukawa matrices, this choice of basis is not preserved with respect to running from $\Lambda$ to the EW scale. As a result, one has to perform back-rotation to the original (Warsaw-down) basis at the EW scale. This process can generate flavour violating semileptonic operators from their flavour conserving counterparts at the scale $\Lambda[9,10,21,62]$. The part of the WET Lagrangian that describes the $b \rightarrow s \ell^{+} \ell^{-}$ processes can be written as

$$
\begin{aligned}
\mathcal{L}_{\mathrm{eff}} & =\frac{\alpha G_{F}}{\sqrt{2} \pi} V_{t b} V_{t s}^{*} \sum_{a=9,10}\left(C_{a} O_{a}+C_{a}^{\prime} O_{a}^{\prime}\right), \\
O_{9(10)}^{i j} & =\left[\bar{s} \gamma_{\mu} P_{L} b\right]\left[\bar{e}_{i} \gamma^{\mu}\left(\gamma_{5}\right) e_{j}\right], \quad O_{9(10)}^{\prime i j}=\left[\bar{s} \gamma_{\mu} P_{R} b\right]\left[\bar{e}_{i} \gamma^{\mu}\left(\gamma_{5}\right) e_{j}\right] .
\end{aligned}
$$

We can match them to the corresponding SMEFT Wilson coefficients as [63]:

$$
\begin{aligned}
C_{9, \mathrm{NP}}^{i j} & =\frac{\pi}{\alpha} \frac{v^{2}}{V_{t b} V_{t s}^{*}}\left(\left[\mathcal{C}_{\ell q}^{(1)}\right]_{i j 23}+\left[\mathcal{C}_{\ell q}^{(3)}\right]_{i j 23}+\left[\mathcal{C}_{q e}\right]_{23 i j}\right), \\
C_{10, \mathrm{NP}}^{i j} & =\frac{\pi}{\alpha} \frac{v^{2}}{V_{t b} V_{t s}^{*}}\left(\left[\mathcal{C}_{q e}\right]_{23 i j}-\left[\mathcal{C}_{\ell q}^{(1)}\right]_{i j 23}-\left[\mathcal{C}_{\ell q}^{(3)}\right]_{i j 23}\right), \\
C_{9, \mathrm{NP}}^{\prime i j} & =\frac{\pi}{\alpha} \frac{v^{2}}{V_{t b} V_{t s}^{*}}\left(\left[\mathcal{C}_{\ell d}\right]_{i j 23}+\left[\mathcal{C}_{e d}\right]_{i j 23}\right) \\
C_{10, \mathrm{NP}}^{\prime i j} & =\frac{\pi}{\alpha} \frac{v^{2}}{V_{t b} V_{t s}^{*}}\left(\left[\mathcal{C}_{e d}\right]_{i j 23}-\left[\mathcal{C}_{\ell d}\right]_{i j 23}\right) .
\end{aligned}
$$

Here, $i j=\mu \mu$,ee. We find that, through back-rotation, the following semileptonic operators can contribute to $b \rightarrow s \ell^{+} \ell^{-}$observables:

$$
\left[\mathcal{C}_{\ell q}^{(1)}\right]_{i j k l},\left[\mathcal{C}_{e d}\right]_{i j k l},\left[\mathcal{C}_{\ell d}\right]_{i j k l},\left[\mathcal{C}_{q e}\right]_{k l i j}
$$


with

$$
i, j, k, l \equiv 1122,1133,2222,2233 .
$$

To constrain these operators we have used the measurements for LFUV observable such as $R_{K^{(*)}}$ [64-70] and $Q_{4,5}=P_{4,5}^{\mu \prime}-P_{4,5}^{e \prime}[71,72]$. However, the full $b \rightarrow s \ell^{+} \ell^{-}$data set can give rise to stronger constraints $[73,74]$.

\subsubsection{Charged current decays}

The $(\mathrm{V}-\mathrm{A}) \times(\mathrm{V}-\mathrm{A})$ type charged-current (c.c) interactions in low energy WET are given by effective Lagrangian

$$
\mathcal{L}_{\text {eff }}^{\text {c.c }}=\left[C_{\nu e d u}^{V, L L}\right]_{i j k l}\left(\bar{\nu}_{i} \gamma_{\mu} P_{L} e_{j}\right)\left(\bar{d}_{k} \gamma_{\mu} P_{L} u_{l}\right)+h . c . .
$$

After the EW symmetry breaking, $\left[C_{\nu e d u}^{V, L L}\right]_{i j k l}$ can be matched with the SMEFT operator $\left[\mathcal{C}_{\ell q}^{(3)}\right]_{i j k l}$. Now at 1-loop level, using (3.18), one can express $\left[C_{\nu e d u}^{V, L L}\right]_{i j k l}$ in terms of high scale Wilson coefficient $\left[\mathcal{C}_{\ell q}^{(1)}\right]_{i j k l}$ due to its mixing with $\left[\mathcal{C}_{\ell q}^{(3)}\right]_{i j k l}$. We find

$$
\left[C_{\nu e d u}^{V, L L}\right]_{i j k l}=6 g_{2}^{2} L\left[\mathcal{C}_{\ell q}^{(1)}\right]_{i j k m}\left(V^{\dagger}\right)_{m l}
$$

Here, the CKM elements $\left(V^{\dagger}\right)_{m l}$ are needed to rotate the up-quark states to the mass basis. The above equation indicates that through EW corrections the singlet operator $\left[\mathcal{C}_{\ell q}^{(1)}\right]_{i j k l}$ can lead to charged current transitions at the low energy. In the following we will look at several charged current processes which can be affected through this mechanism.

$\boldsymbol{K}$ meson decays: at the quark level, the charged-current $K$ decays involve the transition $s \rightarrow u e \bar{\nu}$. Therefore, these decays are driven by the WET operator $\left(\bar{\nu}_{1} \gamma_{\mu} P_{L} e_{1}\right)\left(\bar{d}_{2} \gamma^{\mu} P_{L} u_{1}\right)$. Now, using eq. (4.23), we note that this operator can be generated at the 1-loop level from the high scale SMEFT Wilson coefficient $\left[\mathcal{C}_{\ell q}^{(1)}\right]_{1122}$, i.e.,

$$
\left[C_{\nu e d u}^{V, L L}\right]_{1121} \propto\left(V_{u d}^{*}\left[\mathcal{C}_{\ell q}^{(1)}\right]_{1121}+V_{u s}^{*}\left[\mathcal{C}_{\ell q}^{(1)}\right]_{1122}+V_{u b}^{*}\left[\mathcal{C}_{\ell q}^{(1)}\right]_{1123}\right)
$$

Therefore, at the low-energy, $\left[\mathcal{C}_{\ell q}^{(1)}\right]_{1122}$ can lead to effects in the $K_{L} \rightarrow \pi e \bar{\nu}, K_{S} \rightarrow \pi e \bar{\nu}$, $K^{+} \rightarrow \pi e \bar{\nu}$, and $K^{+} \rightarrow \ell \bar{\nu}$ decays.

$\boldsymbol{\tau} \rightarrow \boldsymbol{K} \boldsymbol{\nu}$ and $\boldsymbol{\tau} \rightarrow \boldsymbol{\pi} \boldsymbol{\nu}$ decays: the $\tau \rightarrow K \nu$ and $\tau \rightarrow \pi \nu$ decays involve $\tau \rightarrow \bar{u} s \nu$ and $\tau \rightarrow \bar{u} d \nu$ transitions, respectively. At the low energy these are governed by the WET operators $\left(\bar{\nu}_{3} \gamma_{\mu} P_{L} e_{3}\right)\left(\bar{d}_{2} \gamma^{\mu} P_{L} u_{1}\right)$ and $\left(\bar{\nu}_{3} \gamma_{\mu} P_{L} e_{3}\right)\left(\bar{d}_{1} \gamma^{\mu} P_{L} u_{1}\right)$. However, at the EW scale these operators can be generated from high scale Wilson coefficients $\left[\mathcal{C}_{\ell q}^{(1)}\right]_{3322}$ and $\left[\mathcal{C}_{\ell q}^{(1)}\right]_{3311}$ respectively (see (4.23)), because

$$
\begin{aligned}
& {\left[C_{\text {vedu }}^{V, L L}\right]_{3321} \propto\left(V_{u d}^{*}\left[\mathcal{C}_{\ell q}^{(1)}\right]_{3321}+V_{u s}^{*}\left[\mathcal{C}_{\ell q}^{(1)}\right]_{3322}+V_{u b}^{*}\left[\mathcal{C}_{\ell q}^{(1)}\right]_{3323}\right)} \\
& {\left[C_{\nu e d u}^{V, L L}\right]_{3311} \propto\left(V_{u d}^{*}\left[\mathcal{C}_{\ell q}^{(1)}\right]_{3311}+V_{u s}^{*}\left[\mathcal{C}_{\ell q}^{(1)}\right]_{3312}+V_{u b}^{*}\left[\mathcal{C}_{\ell q}^{(1)}\right]_{3313}\right) .}
\end{aligned}
$$

Therefore, one can use hadronic $\tau$ decays to constrain the SMEFT operators on the r.h.s. 


\begin{tabular}{|lr|lr|}
\hline Observable & Experimental value & Observable & Experimental value \\
\hline $\mathcal{B}\left(K_{L} \rightarrow \pi e \bar{\nu}\right)$ & $(40.55 \pm 0.11) \times 10^{-2}$ & $\mathcal{B}\left(K_{S} \rightarrow \pi e \bar{\nu}\right)$ & $(7.04 \pm 0.08) \times 10^{-4}$ \\
$\mathcal{B}\left(K^{+} \rightarrow \pi e \bar{\nu}\right)$ & $(5.07 \pm 0.04) \times 10^{-2}$ & $R_{e / \mu}\left(K^{+} \rightarrow \ell \bar{\nu}\right)$ & $(2.488 \pm 0.009) \times 10^{-5}$ \\
\hline $\mathcal{B}(\tau \rightarrow K \nu)$ & $(6.96 \pm 0.10) \times 10^{-3}$ & $\mathcal{B}(\tau \rightarrow \pi \nu)$ & $(10.82 \pm 0.05) \times 10^{-2}$ \\
\hline $\mathcal{B}(\pi \rightarrow e \nu)$ & $(1.2344 \pm 0.0023 \pm 0.0019) \times 10^{-4}$ & & \\
\hline
\end{tabular}

Table 1. The experimental measured values for the charged current $K, \pi$ and $\tau$ decays [76, 77].

$\boldsymbol{\pi} \rightarrow \boldsymbol{e \nu}$ and nuclear $\boldsymbol{\beta}$ decays: the nuclear $\beta$-decay and $\pi \rightarrow e \nu$ are controlled by the WET operator $\left(\bar{\nu}_{1} \gamma_{\mu} P_{L} e_{1}\right)\left(\bar{d}_{1} \gamma^{\mu} P_{L} u_{1}\right)$. From (4.23), clearly at the EW scale this operator can be generated from $\left[\mathcal{C}_{\ell q}^{(1)}\right]_{1111}$ because

$$
\left[C_{\nu e d u}^{V, L L}\right]_{1111} \propto\left(V_{u d}^{*}\left[\mathcal{C}_{\ell q}^{(1)}\right]_{1111}+V_{u s}^{*}\left[\mathcal{C}_{\ell q}^{(1)}\right]_{1112}+V_{u b}^{*}\left[\mathcal{C}_{\ell q}^{(1)}\right]_{1113}\right)
$$

To summarize, we have found that the following list of high scale coefficients can contribute to various charged current decays at low energy:

$$
\left[\mathcal{C}_{\ell q}^{(1)}\right]_{1122},\left[\mathcal{C}_{\ell q}^{(1)}\right]_{3322},\left[\mathcal{C}_{\ell q}^{(1)}\right]_{3311},\left[\mathcal{C}_{\ell q}^{(1)}\right]_{1111}
$$

Note that the Wilson coefficient $\left[\mathcal{C}_{\ell q}^{(3)}\right]_{i j k l}$ also give rise to tree-level contributions to the charged current decays. The experimental measurements for various charged current decays are shown in table 1 . In addition, the measurements for the $\beta$-decays are taken from the ref. [75].

Note that for the charged current operators, in principle the three-body $\tau$ decays such as the Belle spectrum for the process $\tau^{-} \rightarrow K_{S} \pi^{-} \nu_{\tau}$ also apply [78, 79]. But as shown in ref. [80] these decays give rise to similar constraints as $\tau^{-} \rightarrow K \nu$ decay.

\subsubsection{Correlations}

Since a single operator can contribute to several different kinds of observables through RG running, it would be interesting to see how these are correlated to each other. For instance, the operator $\left[\mathcal{C}_{\ell q}^{(1)}\right]_{1111}$ can contribute to $\beta$ decay as well as EWP observables. Similarly, $\left[\mathcal{C}_{\ell q}^{(1)}\right]_{1122}$ contributes to EWP observables, $b \rightarrow s \ell^{+} \ell^{-}$, as well as charged current $K$ processes. Take another example of the operator $\left[\mathcal{C}_{\ell q}^{(3)}\right]_{1111}$, which in addition to $\beta$ decay also contributes to $\pi \rightarrow e \bar{\nu}$ process at tree level. In figure 1, we show correlations between constraints due to various observables in the $\left[\mathcal{C}_{\ell q}^{(1)}\right]_{1111}-\left[\mathcal{C}_{\ell q}^{(1)}\right]_{1122}$ and $\left[\mathcal{C}_{\ell q}^{(1)}\right]_{1111}-\left[\mathcal{C}_{\ell q}^{(3)}\right]_{1111}$ planes in left and right panels respectively. Clearly, in order to get a complete picture about the constrains on a given operator, it is very important to take into account all RG induced observables.

\subsection{Observables for $\Delta F=(1,0)$ operators}

Next, we move on to the semileptonic operators which violate the lepton flavour by one unit and conserve the quark flavour at the scale $\Lambda$. In this case, depending upon the Dirac and flavour structures, both tree-level as well as the 1-loop generated LFV observables 

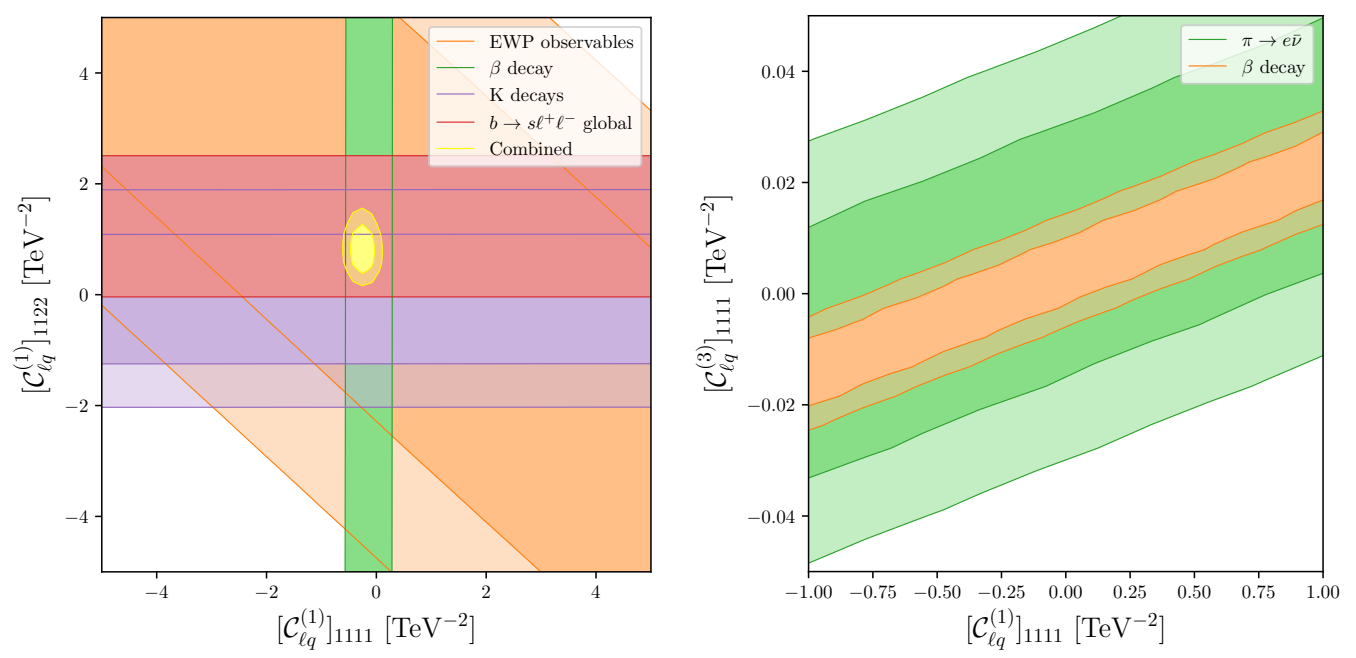

Figure 1. The correlations between various observables in the $\left[\mathcal{C}_{\ell q}^{(1)}\right]_{1111}-\left[\mathcal{C}_{\ell q}^{(1)}\right]_{1122}$ plane (left) and $\left[\mathcal{C}_{\ell q}^{(1)}\right]_{1111}-\left[\mathcal{C}_{\ell q}^{(3)}\right]_{1111}$ plane (right).

are found to be important. The 1-loop generated processes are $\ell_{i} \rightarrow \ell_{j} \bar{\ell} \ell$ and $Z \rightarrow \bar{\ell}_{i} \ell_{j}$ LFV decays. These processes are found to be relevant for all $\Delta F=(1,0)$ operators under consideration. In addition, the $\Delta F=(1,0)$ semileptonic operators can also contribute to $\tau \rightarrow P \ell$ for $P=\pi, \phi$ and $\tau \rightarrow \rho \ell$ processes at the tree-level.

\subsection{1 $Z \rightarrow \bar{\ell}_{i} \ell_{j}$ decays}

The tree-level SMEFT contributions to the LFV $Z$ boson couplings due to $\psi^{2} \phi^{2} D$ operators are given in eqs. (A.1)-(A.7). At the 1-loop level, the semileptonic $\Delta F=(1,0)$ operators can mix with these $\psi^{2} \phi^{2} D$-type operators, as shown in the eqs. (3.20) and (3.19). As a result the $\Delta F=(1,0)$ semileptonic operators can be constrained by the $Z \rightarrow \bar{\ell}_{i} \ell_{j} \mathrm{LFV}$ decays. The current experimental limits on these decays are given in table 2 .

\subsection{2 $\tau \rightarrow 3 \ell$ and $\mu \rightarrow 3 e$ decays}

In WET, the LFV processes such as $\tau \rightarrow 3 \ell$ and $\mu \rightarrow 3 e$ are governed by purely leptonic operators, as given by the effective Lagrangian:

$$
\begin{aligned}
\mathcal{L}_{\text {eff }}^{\text {lloop }}= & {\left[C_{e e}^{V, L L}\right]_{i j l l}\left(\bar{e}_{i} \gamma^{\mu} P_{L} e_{j}\right)\left(\bar{e}_{l} P_{L} \gamma_{\mu} e_{l}\right) } \\
& +\left[C_{e e}^{V, L R}\right]_{i j l l}\left(\bar{e}_{i} \gamma^{\mu} P_{L} e_{j}\right)\left(\bar{e}_{l} \gamma_{\mu} P_{R} e_{l}\right) \\
& +\left[C_{e e}^{V, L R}\right]_{l l i j}\left(\bar{e}_{l} \gamma^{\mu} P_{L} e_{l}\right)\left(\bar{e}_{i} \gamma_{\mu} P_{R} e_{j}\right) \\
& +\left[C_{e e}^{V, R R}\right]_{i j l l}\left(\bar{e}_{i} \gamma^{\mu} P_{R} e_{j}\right)\left(\bar{e}_{l} \gamma_{\mu} P_{R} e_{l}\right)+\text { h.c. }
\end{aligned}
$$

Here, the superscript indicates that such operators are generated only at the 1-loop level and are set to zero at the NP scale. In SMEFT, at tree-level the corresponding four fermion leptonic operators are $\left[\mathcal{C}_{\ell \ell}\right]_{i j k l},\left[\mathcal{C}_{\ell e}\right]_{i j k l}$, and $\left[\mathcal{C}_{e e}\right]_{i j k l}$ which are defined in eqs. (3.11)-(3.13). In addition to this, the SMEFT can also contribute through $\psi^{2} \phi^{2} D$ type operators after integrating out the $Z$ boson. Such contributions arise by combining 
the LFV effective $Z$ boson vertices due to SMEFT with the flavour conserving $Z$ boson interactions in the SM [81]. The $\Delta F=(1,0)$ semileptonic operators can give rise to both effects through operator mixing. From eq. (3.20), one can find that, at the 1-loop level through the EW interactions, the contributing four-fermion SMEFT operators can be directly generated from the semileptonic operators. In addition, the $\psi^{2} \phi^{2} D$ operators get contributions through both the gauge (3.20) as well as the top-Yukawa interactions (3.19). In the LL approximation, combining the four fermion and $\psi^{2} \phi^{2} D$ type contributions, we can express the contributing WET Wilson coefficients at the EW scale directly in terms of SMEFT Wilson coefficients of the semileptonic operators at $\Lambda$ :

$$
\begin{aligned}
{\left[C_{e e}^{V, L L}\right]_{i j l l}=} & \frac{g_{1}^{2}}{3} L\left(-\left[\mathcal{C}_{\ell q}^{(1)}\right]_{i j k k}+\frac{3 g_{2}^{2}}{g_{1}^{2}}\left[\mathcal{C}_{\ell q}^{(3)}\right]_{i j k k}-2\left[\mathcal{C}_{\ell u}\right]_{i j k k}+\left[\mathcal{C}_{\ell d}\right]_{i j k k}\right) \\
& +\frac{\mathcal{Z}}{4}\left(\left[\mathcal{C}_{\ell q}^{(1)}\right]_{i j 33}-\left[\mathcal{C}_{\ell q}^{(3)}\right]_{i j 33}-\left[\mathcal{C}_{\ell u}\right]_{i j 33}\right) g_{L}^{e, \mathrm{SM}} \\
{\left[C_{e e}^{V, L R}\right]_{i j l l}=} & \frac{4 g_{1}^{2}}{3} L\left(-\left[\mathcal{C}_{\ell q}^{(1)}\right]_{i j k k}-2\left[\mathcal{C}_{\ell u}\right]_{i j k k}+\left[\mathcal{C}_{\ell d}\right]_{i j k k}\right) \\
& +\mathcal{Z}\left(\left[\mathcal{C}_{\ell q}^{(1)}\right]_{i j 33}-\left[\mathcal{C}_{\ell q}^{(3)}\right]_{i j 33}-\left[\mathcal{C}_{\ell u}\right]_{i j 33}\right) g_{R}^{e, \mathrm{SM}} \\
{\left[C_{e e}^{V, L R}\right]_{l l i j}=} & \frac{2 g_{1}^{2}}{3} L\left(\left[\mathcal{C}_{e d}^{(1)}\right]_{i j k k}-2\left[\mathcal{C}_{e u}\right]_{i j k k}-\left[\mathcal{C}_{q e}\right]_{k k i j}\right) \\
& +\mathcal{Z}\left(\left[\mathcal{C}_{q e}^{(1)}\right]_{33 i j}-\left[\mathcal{C}_{e u}\right]_{i j 33}\right) g_{L}^{e, \mathrm{SM}}, \\
{\left[C_{e e}^{V, R R}\right]_{i j l l}=} & \frac{2 g_{1}^{2}}{3} L\left(\left[\mathcal{C}_{e d}^{(1)}\right]_{i j k k}-\left[\mathcal{C}_{e u}\right]_{i j k k}-\left[\mathcal{C}_{q e}\right]_{k k i j}\right) \\
& +\frac{\mathcal{Z}}{4}\left(\left[\mathcal{C}_{q e}^{(1)}\right]_{33 i j}-\left[\mathcal{C}_{e u}\right]_{i j 33}\right) g_{R}^{e, S M}
\end{aligned}
$$

with the factor $\mathcal{Z}=\left[3 v^{2} y_{t}^{2} L\right]\left[\frac{g_{Z}^{2}}{M_{Z}^{2}}\right]$. Here $L$ is the $\log$ term defined in (3.16). Numerically, the comparison of the influence of the gauge and Yukawa interactions on the WET Wilson coefficients on the l.h.s. is presented in table 13 in appendix B.4. We find that the following set of Wilson coefficients can be constrained through this mechanism:

$$
\left[\mathcal{C}_{\ell q}^{(1)}\right]_{i j k l},\left[\mathcal{C}_{\ell q}^{(3)}\right]_{i j k l},\left[\mathcal{C}_{e d}\right]_{i j k l},\left[\mathcal{C}_{e u}\right]_{i j k l},\left[\mathcal{C}_{\ell u}\right]_{i j k l},\left[\mathcal{C}_{\ell d}\right]_{i j k l},\left[\mathcal{C}_{q e}\right]_{k l i j},
$$

with

$$
i j k l \equiv 1211,1222,1233,1311,1322,1333,2311,2322,2333 .
$$

The experimental limits used for the LFV decays of $\tau$ and $\mu$ leptons are collected in table 2 .

\subsection{3 $\tau \rightarrow \rho \ell, \tau \rightarrow P(\phi, \pi) \ell$ decays}

In the WET, the hadronic decays $\tau \rightarrow P \ell$ for $P=\pi, \phi$ and $\tau \rightarrow \rho \ell$ can be described by the effective Lagrangian

$$
\begin{aligned}
\mathcal{L}_{\text {eff }}^{\text {tree+1loop }}= & {\left[\mathcal{C}_{e d}^{V, L L}\right]_{i j k k}\left(\bar{e}_{i} \gamma^{\mu} P_{L} e_{j}\right)\left(\bar{d}_{k} \gamma_{\mu} P_{L} d_{k}\right)+\left[\mathcal{C}_{e u}^{V, L L}\right]_{i j 11}\left(\bar{e}_{i} \gamma^{\mu} P_{L} e_{j}\right)\left(\bar{u}_{1} \gamma_{\mu} P_{L} u_{1}\right) } \\
& +\left[\mathcal{C}_{e d}^{V, L R}\right]_{i j k k}\left(\bar{e}_{i} \gamma^{\mu} P_{L} e_{j}\right)\left(\bar{d}_{k} \gamma_{\mu} P_{R} d_{k}\right)+\left[\mathcal{C}_{e u}^{V, L R}\right]_{i j 11}\left(\bar{e}_{i} \gamma^{\mu} P_{L} e_{j}\right)\left(\bar{u}_{1} \gamma_{\mu} P_{R} u_{1}\right) \\
& +\left[\mathcal{C}_{d e}^{V, L R}\right]_{k k i j}\left(\bar{d}_{k} \gamma^{\mu} P_{L} d_{k}\right)\left(\bar{e}_{i} \gamma_{\mu} P_{R} e_{j}\right)+\left[\mathcal{C}_{u e}^{V, L R}\right]_{11 i j}\left(\bar{u}_{1} \gamma^{\mu} P_{L} u_{1}\right)\left(\bar{e}_{i} \gamma_{\mu} P_{R} e_{j}\right) \\
& +\left[\mathcal{C}_{e d}^{V, R R}\right]_{i j k k}\left(\bar{e}_{i} \gamma^{\mu} P_{R} e_{j}\right)\left(\bar{d}_{k} \gamma_{\mu} P_{R} d_{k}\right)+\left[\mathcal{C}_{e u}^{V, R R}\right]_{i j 11}\left(\bar{e}_{i} \gamma^{\mu} P_{R} e_{j}\right)\left(\bar{u}_{1} \gamma_{\mu} P_{R} u_{1}\right) .
\end{aligned}
$$


Here, the indices $k k=11$ for $\tau \rightarrow \pi \ell, \tau \rightarrow \rho \ell$ and 22 for $\tau \rightarrow \phi \ell$. In addition to the tree-level contributions, the semileptonic operators in SMEFT can also contribute to these operators through top-Yukawa loops. Adding the both contributions, in the LL approximation, at the EW scale we obtain:

$$
\begin{aligned}
{\left[\mathcal{C}_{e d}^{V, L L}\right]_{i j k k}=} & {\left[\mathcal{C}_{\ell q}^{(1)}\right]_{i j k k}+\left[\mathcal{C}_{\ell q}^{(3)}\right]_{i j k k} } \\
& +\mathcal{Z}\left[\left[\mathcal{C}_{\ell q}^{(1)}\right]_{i j 33}-\left[\mathcal{C}_{\ell q}^{(3)}\right]_{i j 33}-\left[\mathcal{C}_{\ell u}\right]_{i j 33}\right] g_{L}^{d, \mathrm{SM}}, \\
{\left[\mathcal{C}_{e u}^{V, L L}\right]_{i j 11}=} & V_{1 m}\left[\left[\mathcal{C}_{\ell q}^{(1)}\right]_{i j m n}-\left[\mathcal{C}_{\ell q}^{(3)}\right]_{i j m n}\right] V_{n 1}^{\dagger} \\
& +\mathcal{Z}\left[\left[\mathcal{C}_{\ell q}^{(1)}\right]_{i j 33}-\left[\mathcal{C}_{\ell q}^{(3)}\right]_{i j 33}-\left[\mathcal{C}_{\ell u}\right]_{i j 33}\right] g_{L}^{u, \mathrm{SM}}, \\
{\left[\mathcal{C}_{e d}^{V, L R}\right]_{i j k k}=} & {\left[\mathcal{C}_{\ell d}\right]_{i j k k}+\mathcal{Z}\left[\left[\mathcal{C}_{\ell q}^{(1)}\right]_{i j 33}-\left[\mathcal{C}_{\ell q}^{(3)}\right]_{i j 33}-\left[\mathcal{C}_{\ell u}\right]_{i j 33}\right] g_{R}^{d, \mathrm{SM}}, } \\
{\left[\mathcal{C}_{e u}^{V, L R}\right]_{i j 11}=} & {\left[\mathcal{C}_{\ell u}\right]_{i j 11}+\mathcal{Z}\left[\left[\mathcal{C}_{\ell q}^{(1)}\right]_{i j 33}-\left[\mathcal{C}_{\ell q}^{(3)}\right]_{i j 33}-\left[\mathcal{C}_{\ell u}\right]_{i j 33}\right] g_{R}^{u, \mathrm{SM}}, } \\
{\left[\mathcal{C}_{d e}^{V, L R}\right]_{k k i j}=} & {\left[\mathcal{C}_{q e}\right]_{k k i j}+\mathcal{Z}\left[\left[\mathcal{C}_{q e}\right]_{33 i j}-\left[\mathcal{C}_{e u}\right]_{i j 33}\right] g_{L}^{d, \mathrm{SM}}, } \\
{\left[\mathcal{C}_{u e}^{V, L R}\right]_{11 i j}=} & V_{1 m}\left[\mathcal{C}_{q e}\right]_{m n i j} V_{n 1}^{\dagger}+\mathcal{Z}\left[\left[\mathcal{C}_{q e}\right]_{33 i j}-\left[\mathcal{C}_{e u}\right]_{i j 33}\right] g_{L}^{u, \mathrm{SM}}, \\
{\left[\mathcal{C}_{e d}^{V, R R}\right]_{i j k k}=} & {\left[\mathcal{C}_{e d}\right]_{i j k k}+\mathcal{Z}\left[\left[\mathcal{C}_{q e}\right]_{33 i j}-\left[\mathcal{C}_{e u}\right]_{i j 33}\right] g_{R}^{d, \mathrm{SM}}, } \\
{\left[\mathcal{C}_{e u}^{V, R R}\right]_{i j 11}=} & {\left[\mathcal{C}_{e u}\right]_{i j 11}+\mathcal{Z}\left[\left[\mathcal{C}_{q e}\right]_{33 i j}-\left[\mathcal{C}_{e u}\right]_{i j 33}\right] g_{R}^{u, \mathrm{SM}} . }
\end{aligned}
$$

Here, the CKM elements $V_{i j}$ on the r.h.s. are needed to rotate the up-quarks from the Warsaw-down to the mass basis for matching them with the WET Wilson coefficients on the l.h.s. The quantity $\mathcal{Z}=\left[3 v^{2} y_{t}^{2} L\right]\left[\frac{g_{Z}^{2}}{M_{Z}^{2}}\right]$. Numerically, the relative influence of the WET and SMEFT running can be found in table 14. The following set of Wilson coefficients can be constrained through this mechanism:

$$
\left[\mathcal{C}_{\ell q}^{(1)}\right]_{i j k l},\left[\mathcal{C}_{\ell q}^{(3)}\right]_{i j k l},\left[\mathcal{C}_{e d}\right]_{i j k l},\left[\mathcal{C}_{e u}\right]_{i j k l},\left[\mathcal{C}_{\ell u}\right]_{i j k l},\left[\mathcal{C}_{\ell d}\right]_{i j k l},\left[\mathcal{C}_{q e}\right]_{k l i j}
$$

with

$$
i j k l \equiv 1211,1222,1233,1311,1322,1333,2311,2322,2333 .
$$

Note that the operators involving $u \bar{u}, d \bar{d}$ and $s \bar{s}$ contribute at tree-level, whereas the operators involving the third generation contribute to these processes only at the 1-loop level. Naively, the former contributions are expected to dominate, but we will give a counterexample in the next subsection. The experimental limits on the corresponding LFV decays are shown in table 2 .

\subsubsection{Correlations}

Since, the semileptonic operators can contribute to the LFV processes at tree-level as well as at the 1-loop, it would be interesting to see the relative importance of the loop-level vs. tree-level LFV effects. For example, the Wilson coefficient $\left[\mathcal{C}_{\ell q}^{(1)}\right]_{1311}$ could in principle give a tree level contribution to $\tau \rightarrow P e$ and $\tau \rightarrow \rho e$ processes. However, due to $\mathrm{SU}(2)_{L}$ invariance, the WET Wilson coefficients with $u \bar{u}$ and $d \bar{d}$ flavours get equal contributions on matching with $\left[\mathcal{C}_{\ell q}^{(1)}\right]_{1311}$. As a result, its net effect zero, because the $u \bar{u}$ and $d \bar{d}$ Wilson coefficients 

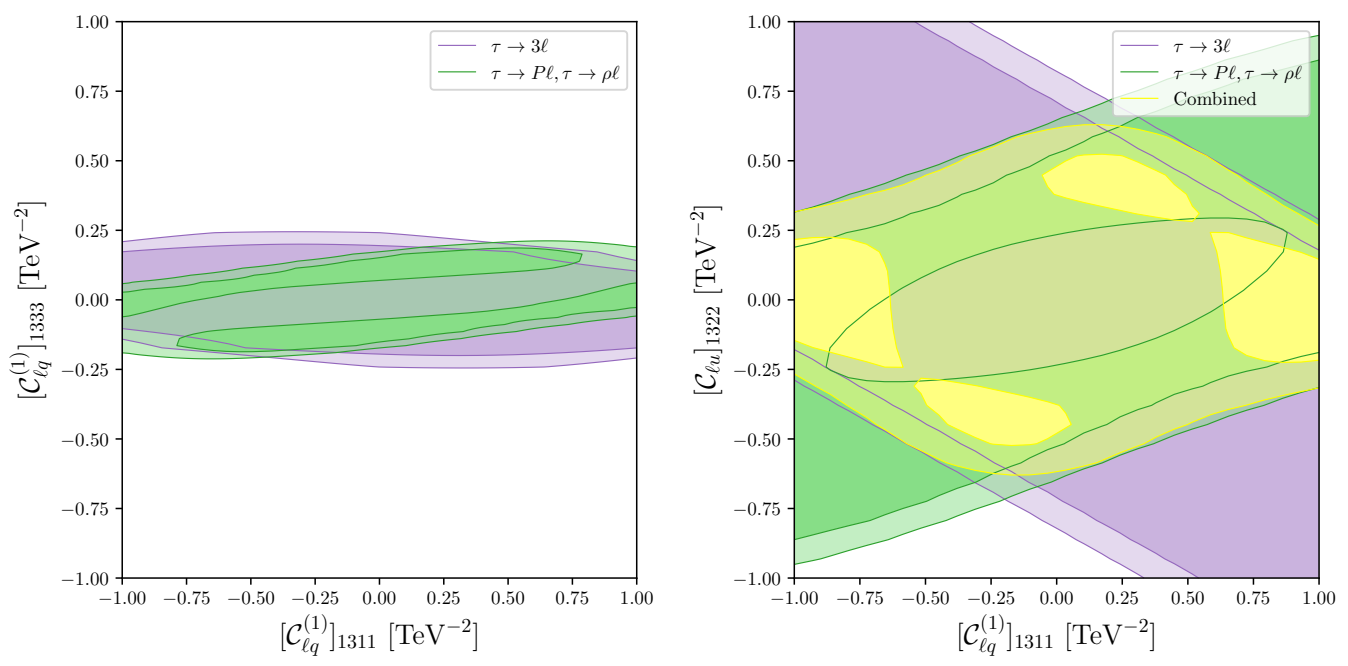

Figure 2. The correlations between various LFV observables in the $\left[\mathcal{C}_{\ell q}^{(1)}\right]_{1311}-\left[\mathcal{C}_{\ell q}^{(1)}\right]_{1333}$ plane (left) and in $\left[\mathcal{C}_{\ell q}^{(1)}\right]_{1311}-\left[\mathcal{C}_{\ell u}\right]_{1322}$ plane (right).

\begin{tabular}{|lr|lr|}
\hline Observable & Experimental value & Observable & Experimental value \\
\hline $\mathcal{B}\left(Z \rightarrow e^{ \pm} \mu^{\mp}\right)$ & $<7.5 \times 10^{-7}[82]$ & $\mathcal{B}\left(Z \rightarrow e^{ \pm} \tau^{\mp}\right)$ & $<5.0 \times 10^{-6}[83]$ \\
$\mathcal{B}\left(Z \rightarrow \mu^{ \pm} \tau^{\mp}\right)$ & $<6.5 \times 10^{-6}[83]$ & & \\
\hline $\mathcal{B}\left(\tau^{-} \rightarrow e^{-} e^{+} e^{-}\right)$ & $<2.7 \times 10^{-8}[84]$ & $\mathcal{B}\left(\tau^{-} \rightarrow \mu^{-} \mu^{+} \mu^{-}\right)$ & $<2.1 \times 10^{-8}[84]$ \\
$\mathcal{B}\left(\tau^{-} \rightarrow e^{-} \mu^{+} \mu^{-}\right)$ & $<2.7 \times 10^{-8}[84]$ & $\mathcal{B}\left(\tau^{-} \rightarrow \mu^{-} e^{+} e^{-}\right)$ & $<1.8 \times 10^{-8}[84]$ \\
$\mathcal{B}\left(\tau^{-} \rightarrow \mu^{-} e^{+} \mu^{-}\right)$ & $<1.7 \times 10^{-8}[84]$ & $\mathcal{B}\left(\tau^{-} \rightarrow e^{-} \mu^{+} e^{-}\right)$ & $<1.5 \times 10^{-8}[84]$ \\
\hline $\mathcal{B}\left(\mu^{-} \rightarrow e^{-} e^{+} e^{-}\right)$ & $<1.0 \times 10^{-12}[85]$ & & \\
\hline $\mathcal{B}(\tau \rightarrow \phi \mu)$ & $<8.4 \times 10^{-8}[86]$ & $\mathcal{B}(\tau \rightarrow \phi e)$ & $<3.1 \times 10^{-8}[86]$ \\
$\mathcal{B}(\tau \rightarrow \rho \mu)$ & $<1.2 \times 10^{-8}[86]$ & $\mathcal{B}(\tau \rightarrow \rho e)$ & $<1.8 \times 10^{-8}[86]$ \\
$\mathcal{B}(\tau \rightarrow \pi \mu)$ & $<1.1 \times 10^{-7}[86]$ & $\mathcal{B}(\tau \rightarrow \pi e)$ & $<8.8 \times 10^{-8}[86]$ \\
\hline
\end{tabular}

Table 2. The experimental upper limits on the LFV decays of $Z$-boson, $\tau$ and $\mu$ leptons. Note only the strongest limits are shown and the upper bounds correspond to 90\% CL for the $Z$ decays and at 90\% CL for all other decays.

enter with opposite sign in the branching ratios (see e.g. eqs. (55) and (61) of ref. [39]). However, $\left[\mathcal{C}_{\ell q}^{(1)}\right]_{1311}$ can still contribute at the 1-loop level through the EW corrections.

On the other hand, the Wilson coefficient $\left[\mathcal{C}_{\ell q}^{(1)}\right]_{1333}$ can not give tree-level contribution to these LFV processes, but it can contribute at 1-loop to $\tau \rightarrow 3 e, \tau \rightarrow e \mu^{+} \mu^{-}$as well as $\tau \rightarrow \rho e$ and $\tau \rightarrow P e$ processes. Interestingly, this loop induced effect on the $u \bar{u}$ and $d \bar{d}$ WET coefficients is not equal but depends on the SM couplings $g_{L}^{d, \mathrm{SM}}$ and $g_{L}^{u, \mathrm{SM}}$ and hence it is not canceled in the branching ratios of our interest (see eqs. (4.37) and (4.38)). In addition to VLL, the VLR (again with unequal sizes for the $u \bar{u}$ and $d \bar{d}$ coefficients) WET operators are also generated at the 1-loop level. In figure 2 (left), the loose constraints 
on $\left[\mathcal{C}_{\ell q}^{(1)}\right]_{1311}$ as compared to $\left[\mathcal{C}_{\ell q}^{(1)}\right]_{1333}$ confirm these findings. On the right panel of the same figure, we show the impact of LFV constraints in the plane of $\left[\mathcal{C}_{\ell q}^{(1)}\right]_{1311}$ and $\left[\mathcal{C}_{\ell u}\right]_{1322}$ Wilson coefficients. In this case, since both operators contribute at the 1-loop level, they are found to be constrained at the similar level.

\section{Sensitivities to the NP scale $\Lambda$}

On the basis of our discussion about the RG running and the various low energy and the EW scale observables identified in the previous sections, now we are in position to look at the highest possible scales for each operator that can be probed using these observables. In eq. (2.1), the Wilson coefficients are defined to be dimensionful quantities. However, these can be written in terms of the dimensionless parameters $\left[c_{X}\right]_{i j k l}$ defined by

$$
\left[\mathcal{C}_{X}\right]_{i j k l}=\frac{\left[c_{X}\right]_{i j k l}}{\Lambda^{2}}
$$

Assuming the presence of a single operator at the scale $\Lambda$, we perform combined fits for each dimensionless parameter $\left[c_{X}\right]_{i j k l}$, using all measurements relevant for a given operator. From this we obtain the quantity $\Lambda / \sqrt{\left[c_{X}\right]_{i j k l}}$ using the central values of $\left[c_{X}\right]_{i j k l}$ from the fits. This gives us a rough estimate of the scales that can be probed for each operator.

Note that in a given NP model, more than one operators can be simultaneously present which could change this simplified picture of single operator dominance. However, the goal of present work is to systematically analyze the low energy implications of each operator separately i.e, to identify the most sensitive observables and assess their potential to constrain the individual operators. In figures $3-9$, we show the central values for the lower bounds on the quantity $\Lambda / \sqrt{\left[c_{X}\right]_{i j k l}}$ (expressed in the units of $\mathrm{TeV}$ ) for various Wilson coefficients under consideration. In the left and right panels, the results for $\Delta F=(0,0)$ and $\Delta F=(1,0)$ operators are shown, respectively. Based on these results, we can now make following important observations:

- The lower bounds on $\Delta F=(0,0)$ operators vary between $\mathcal{O}(\mathrm{TeV})$ to $\mathcal{O}(10 \mathrm{TeV})$, whereas the lower bounds on the $\Delta F=(1,0)$ operators go all the way up to $\mathcal{O}(100 \mathrm{TeV})$, much beyond the present reach of the LHC.

- Due to more stringent experimental limits on the branching ratio for the process $\mu \rightarrow 3 e$ as compared to the $\tau$ LFV modes, the $\Delta F=(1,0)$ operators involving the $12 j j$ flavour indices are very strongly constrained. As shown, the current lower bounds on such operators are always above the ballpark of $10 \mathrm{TeV}$.

- Among $12 j j$ operators, the ones having $j j=33$ are more strongly constrained as compared the operators with $j j=11$ or 22 . This can be attributed to the fact that the former operators can mix strongly with the $\Delta F=(1,0)$ operators $\left[\mathcal{C}_{\phi \ell}^{(1)}\right]_{12}$, $\left[\mathcal{C}_{\phi \ell}^{(3)}\right]_{12}$, and $\left[\mathcal{C}_{\phi e}\right]_{12}$ through top-Yukawa interactions. In this regard, the numerical impact of the top-Yukawa is shown in table 13 and eq. (3.19). Clearly, the Wilson coefficients $\left[\mathcal{C}_{\ell d}\right]_{i j 33}$ and $\left[\mathcal{C}_{e d}\right]_{1233}$ are exceptions here, because they do not mix with the contributing operators through top-Yukawa interactions. 

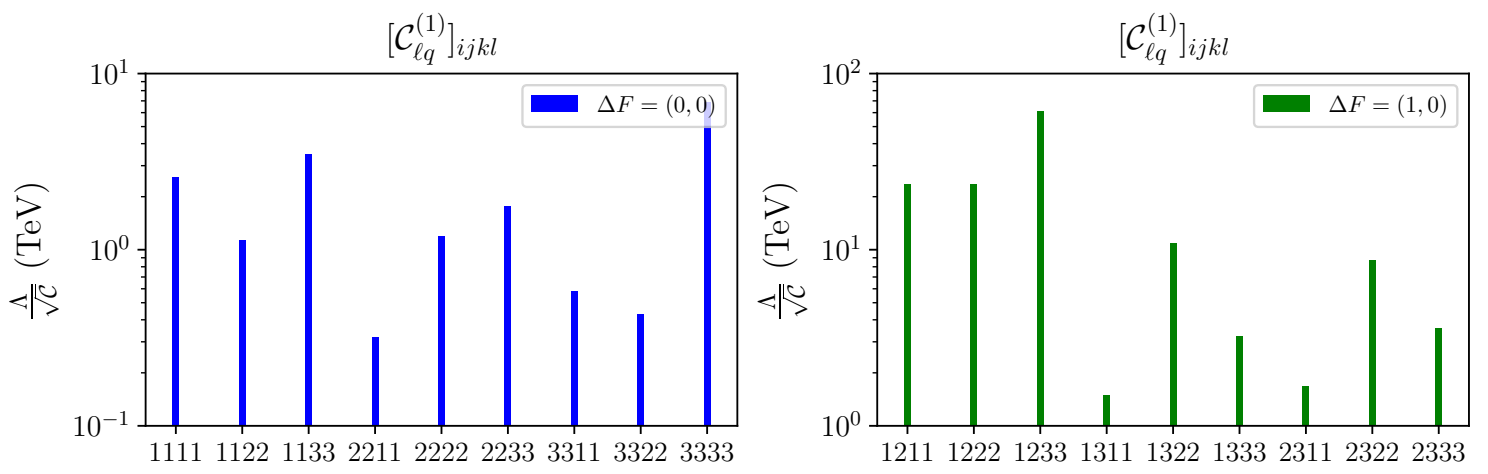

Figure 3. The figure shows the sensitivity of semileptonic operators to the high scale $\Lambda$ normalized with dimensionless parameters $\left[c_{\ell q}^{(1)}\right]_{i j k l}$ as defined in eq. (5.1).

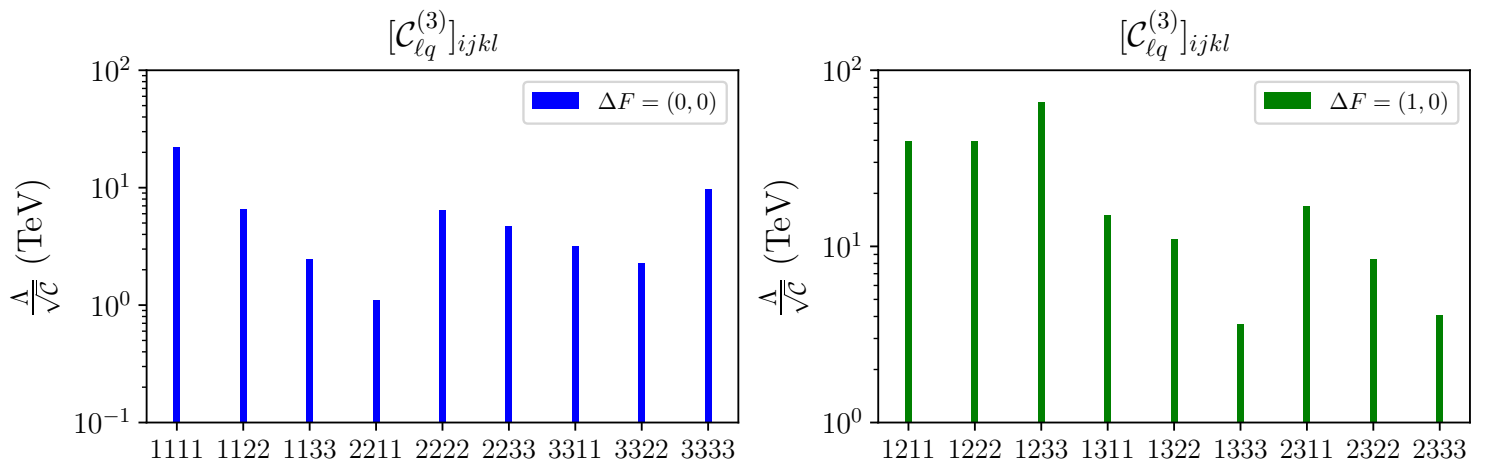

Figure 4. Same as in figure 3 except for $\left[c_{\ell q}^{(3)}\right]_{i j k l}$.

- Since the operators with the indices $13 j j, 23 j j$ contribute even at tree-level to the LFV processes such as $\tau \rightarrow P \ell$ and $\tau \rightarrow \rho \ell$ for $j j=11$ or 22 , as a result these operators are in general more strongly constrained as compared to operators with 1333 or 2333 flavour indices. This is due to the reason that the latter operators contribute only at the 1-loop to such processes or to purely leptonic LFV modes. However, in certain cases like $\left[\mathcal{C}_{\ell q}^{(1)}\right]_{1333},\left[\mathcal{C}_{\ell q}^{(1)}\right]_{2333},\left[\mathcal{C}_{\ell u}\right]_{1333}$ and $\left[\mathcal{C}_{\ell u}\right]_{2333}$, the 1-loop effects due to the top-Yukawa can dominate over the tree-level effects.

- Except for $\left[\mathcal{C}_{\ell d}\right]$ and $\left[\mathcal{C}_{e d}\right]$, the $\Delta F=(0,0)$ operators involving $i i 33$ for $i i=11,22$ or 33 have the strongest bounds, because they contribute to the $Z$ and $W$ boson couplings through the top-Yukawa.

- Finally, among $\Delta F=(0,0)$ operators, $\left[\mathcal{C}_{\ell d}\right]_{i i j j}$ and $\left[\mathcal{C}_{e d}\right]_{i i j j}$ are found to be loosely constrained. In most cases, the lower bound on NP scale lies around $\mathcal{O}(1 \mathrm{TeV})$ or below. This is again due to the fact that these operators do not exhibit the operator mixing due to large top-Yukawa coupling. 

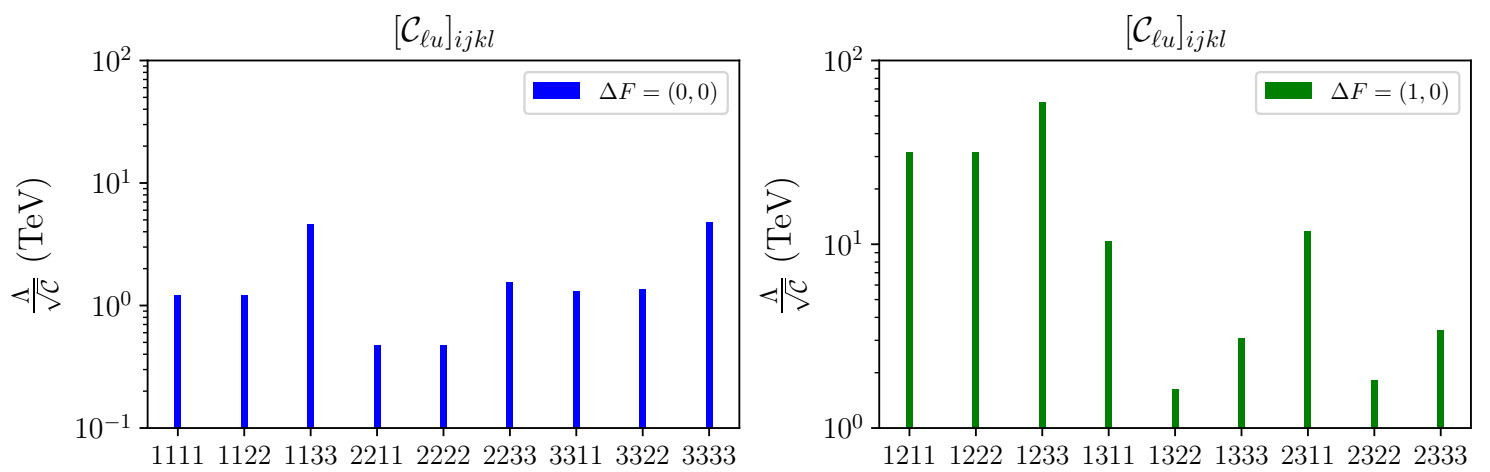

Figure 5. Same as in figure 3 except for $\left[c_{\ell u}\right]_{i j k l}$.
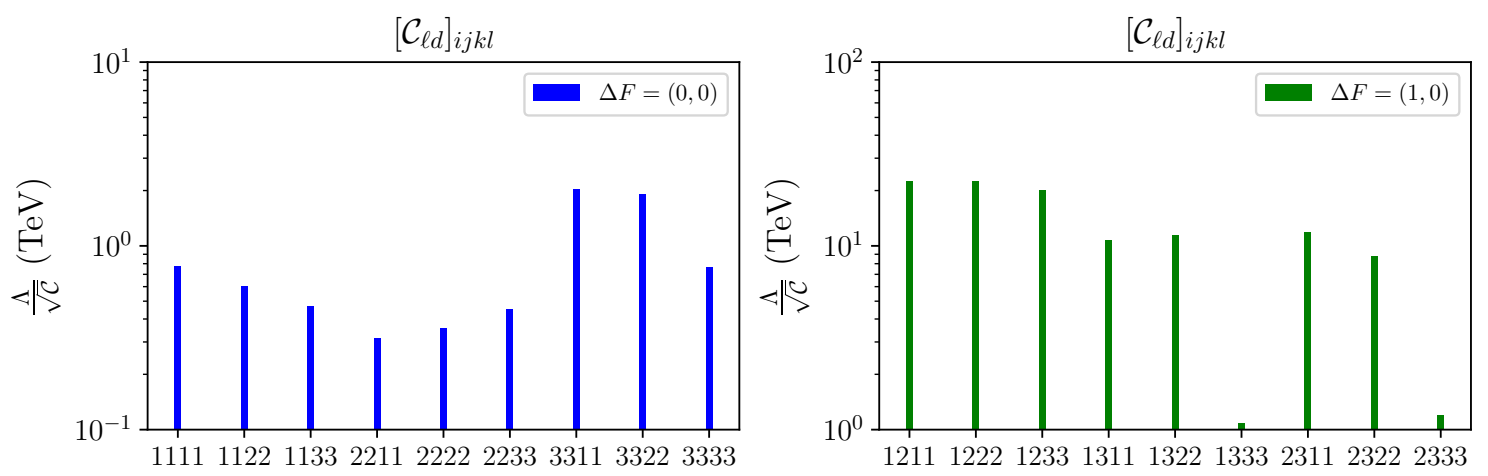

Figure 6. Same as in figure 3 except for $\left[c_{\ell d}\right]_{i j k l}$.
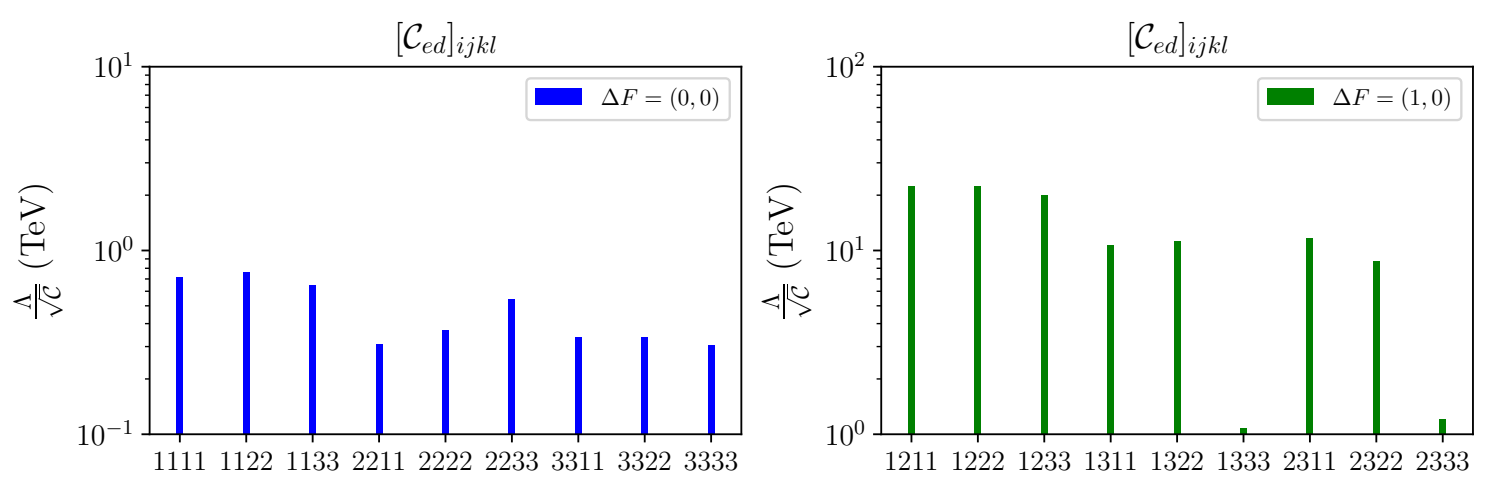

Figure 7. Same as in figure 3 except for $\left[c_{e d}\right]_{i j k l}$. 

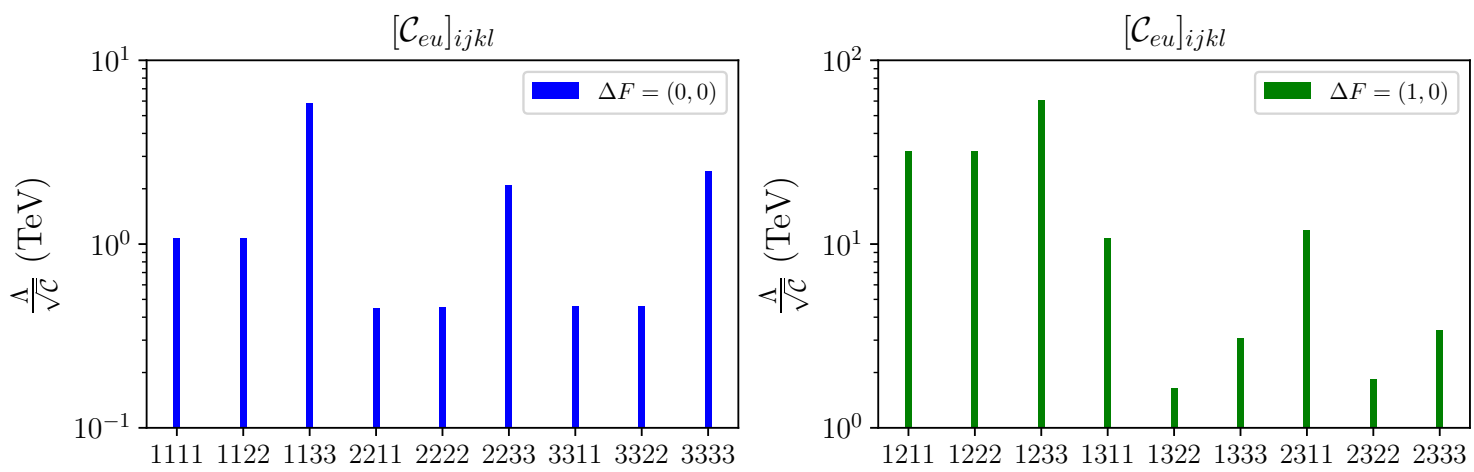

Figure 8. Same as in figure 3 except for $\left[c_{e u}\right]_{i j k l}$.
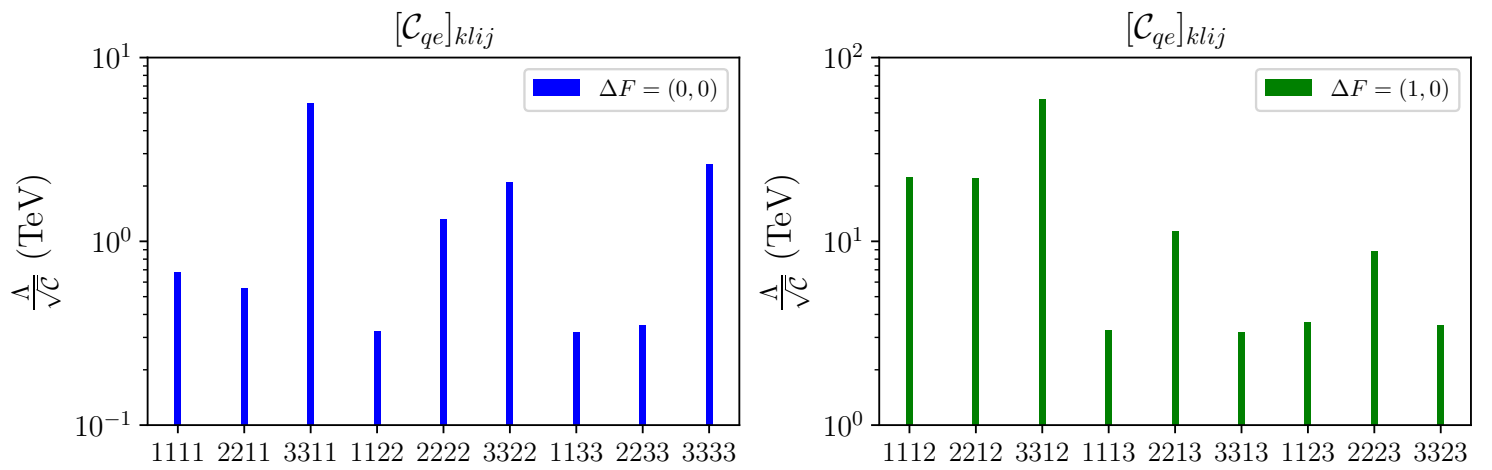

Figure 9. Same as in figure 3 except for $\left[c_{q e}\right]_{i j k l}$.

In appendix $\mathrm{C}$, we also provide the best-fit values along with $1 \sigma$ errors for the dimensionless Wilson coefficients.

\section{Conclusions and outlook}

The SMEFT provides a convenient framework for parameterizing the NP effects beyond the SM. It is well known that the semileptonic operators which violate the quark flavour lead to effects in the flavour violating decays of $B$ and $K$ mesons at low energy, which give rise to stringent constraints. In the view of current anomalies in the $B$-decays, the semileptonic operators are of great interest in general. However, it is important to probe the generic flavour structure of such operators. In particular, often the quark and lepton flavour conserving operators and the ones which violate only the lepton flavour are also generated in the NP models. To probe such operators it is important to know the type of observables to which these operators contribute.

In the present paper, we address this issue. We identify the low energy and the EW scale observables which can be used to probe a generic flavour structure of the semileptonic operators. However, in order to correctly predict the low energy behaviour of these operators, it is necessary to know the operator mixing pattern due to running from NP scale to the EW 
scale and then below. To this end, by scrutinizing the ADMs due to the electroweak gauge as well as the Yukawas interactions one can find that such operators can mix with purely leptonic and $\psi^{2} \phi^{2} D$-type operators in the SMEFT. The former operators can contribute to the LFV decays of leptons, whereas the latter ones in addition also give corrections to the gauge boson couplings at the EW scale.

Therefore, first we have identified the phenomenologically relevant terms in the ADMs and then taking into account the WET and SMEFT RG running effects, we identified a list of observables which can be used to constrain the semileptonic operators having a generic flavour structure. We show that, through SMEFT RG running effects at the 1-loop level, the semileptonic operators can contribute to a variety of observables such as EWP observables, flavour violating decays of $B$ and $K$-mesons - involving neutral as well as charged current transitions, the LFV decays of leptons as well as the LFV Z-boson decays. The main findings of the present study can be summarized as follows:

The semileptonic operators with flavour indices $i i j j$ for $i i, j j=11,22$ or 33 contribute to the EWP observables at the 1-loop level. We have identified two different types of contributions in this context (1) the operator mixing with $\psi^{2} \phi^{2} D$ operators through gauge interactions affect the $W$ and $Z$ boson vertices at the EW scale. Then, through the topYukawa interactions, there are additional contributions from the operators involving third generation in the quark current, i.e., $\left[\mathcal{C}_{\ell q}^{(1)}\right]_{i i 33},\left[\mathcal{C}_{\ell q}^{(3)}\right]_{i i 33},\left[\mathcal{C}_{\ell u}\right]_{i i 33},\left[\mathcal{C}_{e u}\right]_{i i 33}$ and $\left[\mathcal{C}_{q e}\right]_{33 i i}$. (2) The $W$ and $Z$ boson vertices also receive corrections from $\left[\mathcal{C}_{\ell q}^{(3)}\right]_{1133}$ and $\left[\mathcal{C}_{\ell q}^{(3)}\right]_{2233}$ operators via shifts in the $\delta g_{Z}$ and $\delta \sin ^{2} \theta_{W}$. We present the relative impact of the running due to the gauge and top-Yukawas on the $W / Z$ couplings at the EW scale due to semileptonic operators at $\Lambda$. In addition, the quark flavour conserving operators such as, $\left[\mathcal{C}_{\ell q}^{(1)}\right]_{i j k l}$, $\left[\mathcal{C}_{e d}\right]_{i j k l},\left[\mathcal{C}_{\ell d}\right]_{i j k l}$, and $\left[\mathcal{C}_{q e}\right]_{i j k l}$ with $i j k l=1122,1133,2222,2233$ can be constrained by $b \rightarrow s \ell^{+} \ell^{-}$processes through the back-rotation effect. We have shown that the Wilson coefficients $\left[\mathcal{C}_{\ell q}^{(1)}\right]_{i j k l}$ with $i j k l=1122,3322,3311$, or 1111 can contribute to various charged current processes via operator mixing with $\left[\mathcal{C}_{\ell q}^{(3)}\right]_{i j k l}$. This effects goes through the EW corrections.

The semileptonic operators which violate the lepton flavour, while conserving the quark flavour can contribute to the LFV decays of $\tau$ and $\mu$ leptons. Some of the semileptonic operators contribute at tree-level to $\tau \rightarrow P \ell$ and $\tau \rightarrow \rho \ell$ processes. On the other hand, purely leptonic LFV decays such as $\tau \rightarrow 3 \mu$ and $\mu \rightarrow 3 e$ etc., are generated only at the 1-loop level. Again this happens through operator mixing which depends on the gauge as well as Yukawa interactions. In this regard, we find that depending upon the flavour structures the Yukawas play an important in constraining such operators. We have also studied the relative impact of RG running due to various sources on the contributing WET operators at the low scale. These results are presented in table 13 for purely leptonic WET operators and in tables 14 for semileptonic WET operators.

Finally, using the latest measurements, we derived lower bounds on the cut-off scale $\Lambda$ for each semileptonic operator under consideration. We observe that depending upon the flavour structure, the RG induced constraints can lead to sensitivities to very high NP scales varying between $\mathcal{O}(1 \mathrm{TeV})$ to $\mathcal{O}(100 \mathrm{TeV})$. Finally, for a fixed value of $\Lambda=3 \mathrm{TeV}$, we also provide allowed ranges for the semileptonic Wilson coefficients. 
Note added. While finalizing our paper we notice ref. [87] on arXiv which presented a study of the LFV decays through $Z$-flavour violation in the context of future colliders. The discussion on the LFV decays partly overlaps with the present work. However, the aim of the current study is not limited to the LFV modes but is to analyze both flavour conserving as well as the flavour violating processes to which semileptonic operators can contribute at tree-level or through operator mixing.

\section{Acknowledgments}

J.K. thanks Andrzej Buras and Jason Aebischer for carefully reading the manuscript and for the useful comments. J.K. is financially supported by the Alexander von Humboldt Foundation's postdoctoral research fellowship.

\section{A Tree-level shifts in the dim-4 gauge boson couplings}

For completeness, in this section we collect formulae for the tree-level shifts due to SMEFT in the dim- $4 Z$ and $W$ boson couplings at the EW scale. For this purpose we closely follow ref. [49]. The shifts in the $Z$ boson couplings $\delta\left(g_{X}^{Y}\right)_{i j}^{\text {dir }}$ (see eq. (4.1) for definition) get tree-level contributions due to $\psi^{2} \phi^{2} D$-type operators in the SMEFT. At the EW scale for the quarks these are given by

$$
\begin{aligned}
\delta\left(g_{L}^{u}\right)_{i j}^{\operatorname{dir}} & =-\frac{v^{2}}{2} V_{i m}\left(\left[\mathcal{C}_{\phi q}^{(1)}\right]_{m n}-\left[\mathcal{C}_{\phi q}^{(3)}\right]_{m n}\right) V_{n j}^{\dagger} \\
\delta\left(g_{R}^{u}\right)_{i j}^{\operatorname{dir}} & =-\frac{v^{2}}{2}\left[\mathcal{C}_{\phi u}\right]_{i j} \\
\delta\left(g_{L}^{d}\right)_{i j}^{\text {dir }} & =-\frac{v^{2}}{2}\left(\left[\mathcal{C}_{\phi q}^{(1)}\right]_{i j}+\left[\mathcal{C}_{\phi q}^{(3)}\right]_{i j}\right) \\
\delta\left(g_{R}^{d}\right)_{i j}^{\text {dir }} & =-\frac{v^{2}}{2}\left[\mathcal{C}_{\phi d}\right]_{i j} .
\end{aligned}
$$

Similarly, for the leptons we have

$$
\begin{aligned}
& \delta\left(g_{L}^{\nu}\right)_{i j}^{\operatorname{dir}}=-\frac{v^{2}}{2}\left(\left[\mathcal{C}_{\phi \ell}^{(1)}\right]_{i j}-\left[\mathcal{C}_{\phi \ell}^{(3)}\right]_{i j}\right), \\
& \delta\left(g_{L}^{e}\right)_{i j}^{\operatorname{dir}}=-\frac{v^{2}}{2}\left(\left[\mathcal{C}_{\phi \ell}^{(1)}\right]_{i j}+\left[\mathcal{C}_{\phi \ell}^{(3)}\right]_{i j}\right), \\
& \delta\left(g_{R}^{e}\right)_{i j}^{\operatorname{dir}}=-\frac{v^{2}}{2}\left[\mathcal{C}_{\phi e}\right]_{i j} .
\end{aligned}
$$

The CKM elements appearing in the expressions for $\delta\left(g_{L}^{u}\right)_{i j}^{\text {dir }}$ are needed due to our Warsawdown basis choice for the SMEFT operators. In addition, the shifts in the parameters $g_{Z}$ and $\sin \theta_{W}$ are given by

$$
\delta g_{Z}=-\frac{v^{2}}{2}\left(\left[\mathcal{C}_{\phi \ell}^{(3)}\right]_{11}+\left[\mathcal{C}_{\phi \ell}^{(3)}\right]_{22}-\frac{\left[\mathcal{C}_{\ell \ell}\right]_{1221}}{2}\right)
$$

and

$$
\delta \sin ^{2} \theta_{W}=\frac{v^{2} \sin ^{2} 2 \theta_{W}}{4 \cos 2 \theta_{W}}\left(\left[\mathcal{C}_{\phi \ell}^{(3)}\right]_{11}+\left[\mathcal{C}_{\phi \ell}^{(3)}\right]_{22}-\frac{\left[\mathcal{C}_{\ell \ell}\right]_{1221}}{2}\right)
$$




\begin{tabular}{|rrr|}
\hline Coupling $\left(m_{Z}\right)$ & Gauge Couplings & Yukawa Couplings \\
\hline$\left[\mathcal{C}_{\ell q}^{(1)}\right]_{i i 33}(\Lambda)=1$ & & \\
$\delta\left(g_{L}^{u}\right)_{11}$ & - & 2.28 \\
$\delta\left(g_{L}^{u}\right)_{22}$ & - & 2.28 \\
$\delta\left(g_{L}^{d}\right)_{11}$ & - & -1.68 \\
$\delta\left(g_{L}^{d}\right)_{22}$ & -4.02 & -1.68 \\
$\delta\left(g_{L}^{d}\right)_{33}$ & 3.58 & -5.67 \\
$\delta\left(g_{L}^{e}\right)_{i i}$ & - & 220.63 \\
$\delta\left(g_{L}^{e}\right)_{j j}$ & - & -2.87 \\
$\delta\left(g_{R}^{u}\right)_{11}$ & - & 1.19 \\
$\delta\left(g_{R}^{u}\right)_{22}$ & - & 1.19 \\
$\delta\left(g_{R}^{e}\right)_{k k}$ & & -1.79 \\
\hline
\end{tabular}

Table 3. The shifts in the $Z$ boson couplings with fermions i.e., $\delta\left(g_{X}^{Y}\right)_{i i} \times 10^{5}$ at $\mu \simeq 91 \mathrm{GeV}$ due to semileptonic Wilson coefficient $\left[\mathcal{C}_{\ell q}^{(1)}\right]_{i i 33}(\Lambda)=1 \mathrm{TeV}^{-2}$ at $\Lambda=1 \mathrm{TeV}$. Here $i i=11$ or 22 , $j j=11,22,33 \neq i i$ and $k k=11,22,33$. In the second and third column, the RG running due to gauge interactions only and gauge + Yukawa interactions is shown, respectively. Note, only the non-zero entries are shown.

The $W$ boson couplings can be analogously parameterized as

$$
\begin{aligned}
& \delta\left(\varepsilon_{L}^{\ell}\right)_{i j}^{\operatorname{dir}}=v^{2}\left[\mathcal{C}_{\phi \ell}^{(3)}\right]_{i j}, \\
& \delta\left(\varepsilon_{L}^{q}\right)_{i j}^{\operatorname{dir}}=v^{2} V_{i m}\left[\mathcal{C}_{\phi q}^{(3)}\right]_{m j} .
\end{aligned}
$$

Since, we are interested to study the effects of only the semileptonic operators on the EWP observables, we have ignored additional corrections due to $\left[\mathcal{C}_{\phi D}\right]$ and $\left[\mathcal{C}_{\phi W B}\right]$ operators [49]. In this regard, we have checked that these operators can not be generated from semileptonic operators via operator mixing.

\section{B RG induced shifts in the dim-4 and dim-6 operators}

Depending upon the scale and the interactions involved, there are three types of RGEs, i.e., due to the gauge and Yukawa interactions in the SMEFT and due to QCD+QED interactions in the WET. In this section, we analyze the relative impact of three different types of RG runnings on the dim- 4 and dim- 6 operators which contribute to the EWP observables and the LFV processes at the low energy.

\section{B.1 Dim-4 $Z$ boson couplings}

In table $3-10$, we show the $Z$ and $W$ boson gauge couplings at the EW scale for a given semileptonic operator introduced at the high scale. 


\begin{tabular}{|c|c|c|}
\hline Coupling $\left(m_{Z}\right)$ & Gauge Couplings & Yukawa Couplings \\
\hline$\left[\mathcal{C}_{\ell q}^{(1)}\right]_{3333}(\Lambda)=1$ & & \\
\hline$\delta\left(g_{L}^{d}\right)_{33}$ & -4.04 & -4.27 \\
\hline$\delta\left(g_{L}^{e}\right)_{33}$ & 3.54 & 222.69 \\
\hline
\end{tabular}

Table 4. Same as in table 3 except for $\left[\mathcal{C}_{\ell q}^{(1)}\right]_{3333}(\Lambda)$.

\begin{tabular}{|c|c|c|}
\hline Coupling $\left(m_{Z}\right)$ & Gauge Couplings & Yukawa Couplings \\
\hline$\left[\mathcal{C}_{\ell q}^{(3)}\right]_{i i 33}(\Lambda)=1$ & & \\
\hline$\delta\left(g_{L}^{u}\right)_{11}$ & - & -181.11 \\
\hline$\delta\left(g_{L}^{u}\right)_{22}$ & - & -181.11 \\
\hline$\delta\left(g_{L}^{d}\right)_{11}$ & - & 147.73 \\
\hline$\delta\left(g_{L}^{d}\right)_{22}$ & - & 147.73 \\
\hline$\delta\left(g_{L}^{d}\right)_{33}$ & 12.86 & 160.61 \\
\hline$\delta\left(g_{L}^{e}\right)_{i i}$ & 38.66 & 17.72 \\
\hline$\delta\left(g_{L}^{e}\right)_{j j}$ & - & 214.52 \\
\hline$\delta\left(g_{R}^{u}\right)_{11}$ & - & -66.79 \\
\hline$\delta\left(g_{R}^{u}\right)_{22}$ & - & -66.79 \\
\hline$\delta\left(g_{R}^{d}\right)_{k k}$ & - & 33.40 \\
\hline$\delta\left(g_{R}^{e}\right)_{k k}$ & - & 100.19 \\
\hline
\end{tabular}

Table 5. Same as in table 3 except for $\left[\mathcal{C}_{\ell q}^{(3)}\right]_{i i 33}(\Lambda)$ with $i i=11$ or 22.

\begin{tabular}{|c|c|c|}
\hline Coupling $\left(m_{Z}\right)$ & Gauge Couplings & Yukawa Couplings \\
\hline$\left[\mathcal{C}_{\ell q}^{(3)}\right]_{3333}(\Lambda)=1$ & & \\
\hline$\delta\left(g_{L}^{d}\right)_{33}$ & 12.93 & 12.61 \\
\hline$\delta\left(g_{L}^{e}\right)_{33}$ & 38.76 & -197.61 \\
\hline
\end{tabular}

Table 6. Same as in table 3 except for $\left[\mathcal{C}_{\ell q}^{(3)}\right]_{3333}(\Lambda)$.

\begin{tabular}{|rrr|}
\hline Coupling $\left(m_{Z}\right)$ & Gauge Couplings & Yukawa Couplings \\
\hline$\left[\mathcal{C}_{\ell u}\right]_{i i 33}(\Lambda)=1$ & & \\
$\delta\left(g_{L}^{e}\right)_{i i}$ & 7.85 & -208.36 \\
\hline
\end{tabular}

Table 7 . Same as in table 3 except for $\left[\mathcal{C}_{\ell u}\right]_{i i 33}(\Lambda)$ with $i i=11,22$ or 33. 


\begin{tabular}{|rrr|}
\hline Coupling $\left(m_{Z}\right)$ & Gauge Couplings & Yukawa Couplings \\
\hline$\left[\mathcal{C}_{\ell d}\right]_{i i 33}(\Lambda)=1$ & & \\
$\delta\left(g_{L}^{e}\right)_{i i}$ & -3.95 & -3.89 \\
$\delta\left(g_{R}^{d}\right)_{33}$ & -3.92 & -3.92 \\
\hline
\end{tabular}

Table 8. Same as in table 3 except for $\left[\mathcal{C}_{\ell d}\right]_{i i 33}(\Lambda)$ with $i i=11,22$ or 33.

\begin{tabular}{|rrr|}
\hline Coupling $\left(m_{Z}\right)$ & Gauge Couplings & Yukawa Couplings \\
\hline$\left[\mathcal{C}_{e u}\right]_{i i 33}(\Lambda)=1$ & & \\
$\delta\left(g_{R}^{e}\right)_{i i}$ & 7.81 & -211.10 \\
\hline
\end{tabular}

Table 9. Same as in table 3 except for $\left[\mathcal{C}_{e u}\right]_{i i 33}(\Lambda)$ with $i i=11,22$ or 33.

\begin{tabular}{|c|c|c|}
\hline Coupling $\left(m_{Z}\right)$ & Gauge Couplings & Yukawa Couplings \\
\hline$\left[\mathcal{C}_{q e}^{(1)}\right]_{33 i i}(\Lambda)=1$ & & \\
\hline$\delta\left(g_{L}^{d}\right)_{33}$ & -3.91 & -4.13 \\
\hline$\delta\left(g_{R}^{e}\right)_{i i}$ & 3.87 & 220.04 \\
\hline
\end{tabular}

Table 10. Same as in table 3 except for $\left[\mathcal{C}_{q e}\right]_{33 i i}(\Lambda)$ with $i i=11,22$ or 33.

\begin{tabular}{|c|c|c|}
\hline Coupling $\left(m_{W}\right)$ & Gauge Couplings & Yukawa Couplings \\
\hline$\left[\mathcal{C}_{\ell q}^{(3)}\right]_{i i 33}(\Lambda)=1$ & & \\
\hline$\delta\left(\varepsilon_{L}^{q}\right)_{11}$ & - & -328.37 \\
\hline$\delta\left(\varepsilon_{L}^{q}\right)_{22}$ & - & -328.37 \\
\hline$\delta\left(\varepsilon_{L}^{\ell}\right)_{i i}$ & -77.31 & 52.11 \\
\hline$\delta\left(\varepsilon_{L}^{\ell}\right)_{j j}$ & - & -328.37 \\
\hline
\end{tabular}

Table 11. The shifts in the $W$ boson couplings i.e., $\delta\left(\varepsilon_{L}^{Y}\right)_{i i} \times 10^{5}$ to fermions at $\mu \simeq 91 \mathrm{GeV}$ due to semileptonic operator $\left[\mathcal{C}_{\ell q}^{(3)}\right]_{i i 33}(\Lambda)=1 \mathrm{TeV}^{-2}$ at $\Lambda=1 \mathrm{TeV}$. Here $i i=11$ or 22 , and $j j=11,22,33 \neq i i$. In the second and third column the RG running due to gauge only and gauge + Yukawa interactions is included, respectively. Note, only the non-zero entries are shown.

\section{B.2 Dim-4 $W$ boson couplings}

In table 11 and 12 we show impact of RG running due to Yukawas on the $W$ couplings to fermions at the EW scale.

\section{B.3 Leptonic dim-6 WET operators}

In table 13, we present the numerical values for the leptonic WET Wilson coefficients by setting the SMEFT Wilson coefficients to $1 \mathrm{TeV}^{-2}$ at $\Lambda=1 \mathrm{TeV}$. In order to study the roles played by different kinds of RG effects, we present three kind of numbers, (1) with only WET 


\begin{tabular}{|rrr|}
\hline Coupling $\left(m_{W}\right)$ & Gauge Couplings & Yukawa Couplings \\
\hline$\left[\mathcal{C}_{\ell q}^{(3)}\right]_{3333}(\Lambda)=1$ & & \\
$\delta\left(\varepsilon_{L}^{\ell}\right)_{33}$ & -77.46 & 381.44 \\
\hline
\end{tabular}

Table 12. Same as in table 11 except for $\left[\mathcal{C}_{\ell q}^{(3)}\right]_{3333}(\Lambda)$.

(QED+QCD) running, (2) WET + SMEFT running due to gauge interactions, and (3) the full WET + SMEFT gauge + Yukawa running. As we can see, except for $\left[\mathcal{C}_{\ell d}\right]_{1333}$ and $\left[\mathcal{C}_{e d}\right]_{1333}$, the top-Yukawa effects always play an important role for the operators involving third generation quarks.

\section{B.4 Semileptonic dim-6 WET operators}

In table 14, we present the low energy semileptonic WET Wilson coefficients by setting various semileptonic SMEFT Wilson coefficients, involving the third generation of quarks, equal to $1 \mathrm{TeV}^{-2}$ at $\Lambda=1 \mathrm{TeV}$. Again, in order to study the roles played by different kinds of RG running, we have presented three kind of numbers. As found in the case of WET leptonic operators, except for the Wilson coefficients $\left[\mathcal{C}_{\ell d}\right]_{1333}$ and $\left[\mathcal{C}_{e d}\right]_{1333}$, the top-Yukawa always plays an important role.

\section{Bounds on the Wilson coefficients}

In this section, we report the allowed ranges for the semileptonic Wilson coefficients $\left[c_{X}\right]_{i j k l}$ which are defined in (5.1). Considering various 1-loop induced constraints, as discussed in section 4 , the allowed values of the dimensionless parameters $\left[c_{X}\right]_{i j k l}$ are obtained by performing the fits. The results are presented in tables 15-21. The uncertainties are also indicated at $1 \sigma$ level. In all cases, the cut-off scale $\Lambda$ is set to $3 \mathrm{TeV}$. 


\begin{tabular}{|c|c|c|c|}
\hline Coefficent & $\mathrm{QCD}+\mathrm{QED}$ & Gauge Couplings & Yukawa Couplings \\
\hline \multicolumn{4}{|l|}{$\left[\mathcal{C}_{\ell q}^{(1)}\right]_{1311}(\Lambda)=1$} \\
\hline$\left[C_{e e}^{V, L L}\right]_{\ell \ell 13}$ & 3.41 & 5.48 & 5.48 \\
\hline$\left[C_{e e}^{V, L R}\right]_{13 \ell \ell}$ & 3.41 & 5.64 & 5.63 \\
\hline \multicolumn{4}{|l|}{$\left[\mathcal{C}_{\ell q}^{(1)}\right]_{1333}(\Lambda)=1$} \\
\hline$\left[C_{e e}^{V, L L}\right]_{\ell \ell 13}$ & -3.40 & -1.49 & -35.25 \\
\hline$\left[C_{e e}^{V, L R}\right]_{13 \ell \ell}$ & -3.40 & -1.33 & 36.98 \\
\hline \multicolumn{4}{|l|}{$\left[\mathcal{C}_{\ell q}^{(3)}\right]_{1311}(\Lambda)=1$} \\
\hline$\left[C_{e e}^{V, L L}\right]_{\ell \ell 13}$ & -10.24 & -15.91 & 15.91 \\
\hline$\left[C_{e e}^{V, L R}\right]_{13 \ell \ell}$ & -10.24 & -16.66 & 16.66 \\
\hline \multicolumn{4}{|l|}{$\left[\mathcal{C}_{\ell q}^{(3)}\right]_{1333}(\Lambda)=1$} \\
\hline$\left[C_{e e}^{V, L L}\right]_{\ell \ell 13}$ & -3.42 & -8.42 & 47.68 \\
\hline$\left[C_{e e}^{V, L R}\right]_{13 \ell \ell}$ & -3.42 & -9.17 & -29.10 \\
\hline \multicolumn{4}{|l|}{$\left[\mathcal{C}_{\ell u}\right]_{1311}(\Lambda)=1$} \\
\hline$\left[C_{e e}^{V, L L}\right]_{\ell \ell 13}$ & 6.83 & 10.37 & 10.37 \\
\hline$\left[C_{e e}^{V, L R}\right]_{13 \ell \ell}$ & 6.83 & 10.67 & 10.67 \\
\hline \multicolumn{4}{|l|}{$\left[\mathcal{C}_{\ell u}\right]_{1333}(\Lambda)=1$} \\
\hline$\left[C_{e e}^{V, L L}\right]_{\ell \ell 13}$ & - & 3.60 & -39.64 \\
\hline$\left[C_{e e}^{V, L R}\right]_{13 \ell \ell}$ & - & 3.90 & -39.08 \\
\hline \multicolumn{4}{|l|}{$\left[\mathcal{C}_{\ell d}\right]_{1311}(\Lambda)=1$} \\
\hline$\left[C_{e e}^{V, L L}\right]_{\ell \ell 13}$ & -3.14 & -5.24 & 5.24 \\
\hline$\left[C_{e e}^{V, L R}\right]_{13 \ell \ell}$ & -3.14 & -5.39 & 5.39 \\
\hline \multicolumn{4}{|l|}{$\left[\mathcal{C}_{\ell d}\right]_{1333}(\Lambda)=1$} \\
\hline$\left[C_{e e}^{V, L L}\right]_{\ell \ell 13}$ & -3.14 & -5.24 & 5.23 \\
\hline$\left[C_{e e}^{V, L R}\right]_{13 \ell \ell}$ & -3.14 & -5.39 & 5.40 \\
\hline \multicolumn{4}{|l|}{$\left[\mathcal{C}_{e u}\right]_{1311}(\Lambda)=1$} \\
\hline$\left[C_{e e}^{V, L R}\right]_{\ell \ell 13}$ & 6.83 & 10.88 & -10.88 \\
\hline$\left[C_{e e}^{V, R R}\right]_{\ell \ell 13}$ & 6.83 & 10.88 & -10.88 \\
\hline \multicolumn{4}{|l|}{$\left[\mathcal{C}_{e u}\right]_{1333}(\Lambda)=1$} \\
\hline$\left[C_{e e}^{V, L R}\right]_{\ell \ell 13}$ & - & 3.94 & -35.65 \\
\hline$\left[C_{e e}^{V, R R}\right]_{\ell \ell 13}$ & - & 3.94 & -35.65 \\
\hline \multicolumn{4}{|l|}{$\left[\mathcal{C}_{e d}\right]_{1311}(\Lambda)=1$} \\
\hline$\left[C_{e e}^{V, L R}\right]_{\ell \ell 13}$ & -3.14 & -5.33 & 5.33 \\
\hline$\left[C_{e e}^{V, R R}\right]_{\ell \ell 13}$ & -3.14 & -5.33 & 5.33 \\
\hline \multicolumn{4}{|l|}{$\left[\mathcal{C}_{e d}\right]_{1333}(\Lambda)=1$} \\
\hline$\left[C_{e e}^{V, L R}\right]_{\ell \ell 13}$ & -3.14 & -5.33 & -5.32 \\
\hline$\left[C_{e e}^{V, R R}\right]_{\ell \ell 13}$ & -3.14 & -5.33 & -5.32 \\
\hline \multicolumn{4}{|l|}{$\left[\mathcal{C}_{q e}\right]_{1311}(\Lambda)=1$} \\
\hline$\left[C_{e e}^{V, L R}\right]_{\ell \ell 13}$ & 3.41 & 5.35 & -5.35 \\
\hline$\left[C_{e e}^{V, R R}\right]_{\ell \ell 13}$ & 3.41 & 5.35 & -5.35 \\
\hline \multicolumn{4}{|l|}{$\left[\mathcal{C}_{q e}\right]_{1333}(\Lambda)=1$} \\
\hline$\left[C_{e e}^{V, L R}\right]_{\ell \ell 13}$ & -3.4 & -1.44 & -37.66 \\
\hline$\left[C_{e e}^{V, R R}\right]_{\ell \ell 13}$ & -3.400 & -1.44 & -37.66 \\
\hline
\end{tabular}

Table 13. The impact of semileptonic operators on the low energy purely leptonic $\Delta F=(1,0)$ Wilson coefficients (in $10^{-9} \mathrm{TeV}^{-2}$ units) at $\mu_{\text {low }}=2 \mathrm{GeV}$ is shown. The second column refers to the values with only WET $(\mathrm{QED}+\mathrm{QCD})$ running, the third column refers to the WET + SMEFT running due to gauge interactions, and the fourth column refers to the full WET+ SMEFT gauge + Yukawa running. Here, the indices $\ell \ell=11,22$, or 33 and $\Lambda=1 \mathrm{TeV}$. The SMEFT Wilson coefficients are set to 1 (in $\mathrm{TeV}^{-2}$ units) at $\Lambda$. 


\begin{tabular}{|c|c|c|c|}
\hline Coefficent & $\mathrm{QCD}+\mathrm{QED}$ & Gauge Couplings & Yukawa Couplings \\
\hline \multicolumn{4}{|l|}{$\left[\mathcal{C}_{\ell q}^{(1)}\right]_{1333}(\Lambda)=1$} \\
\hline$\left[C_{e d}^{V, L L}\right]_{13 j j}$ & -1.13 & -0.47 & -59.92 \\
\hline$\left[C_{e d}^{V, L R}\right]_{13 j j}$ & -1.13 & -0.46 & 12.11 \\
\hline$\left[C_{e u}^{V, L L}\right]_{1311}$ & 2.28 & 0.92 & 49.59 \\
\hline$\left[C_{e u}^{V, L R}\right]_{1311}$ & 2.27 & 0.97 & -23.60 \\
\hline \multicolumn{4}{|l|}{$\left[\mathcal{C}_{\ell q}^{(3)}\right]_{1333}(\Lambda)=1$} \\
\hline$\left[C_{e d}^{V, L L}\right]_{13 j j}$ & -1.14 & -2.63 & 67.29 \\
\hline$\left[C_{e d}^{V, L R}\right]_{13 j j}$ & -1.14 & -3.02 & -9.50 \\
\hline$\left[C_{e u}^{V, L L}\right]_{1311}$ & 2.27 & 6.44 & -60.48 \\
\hline$\left[C_{e u}^{V, L R}\right]_{1311}$ & 2.28 & 5.91 & 18.38 \\
\hline \multicolumn{4}{|l|}{$\left[\mathcal{C}_{\ell u}\right]_{1333}(\Lambda)=1$} \\
\hline$\left[C_{e d}^{V, L L}\right]_{13 j j}$ & - & 1.24 & -58.33 \\
\hline$\left[C_{e d}^{V, L R}\right]_{13 j j}$ & - & 1.27 & 12.77 \\
\hline$\left[C_{e u}^{V, L L}\right]_{1311}$ & - & -2.56 & 47.26 \\
\hline$\left[C_{e u}^{V, L R}\right]_{1311}$ & - & -2.43 & -24.76 \\
\hline \multicolumn{4}{|l|}{$\left[\mathcal{C}_{\ell d}\right]_{1333}(\Lambda)=1$} \\
\hline$\left[C_{e d}^{V, L L}\right]_{13 j j}$ & -1.14 & -1.77 & 1.75 \\
\hline$\left[C_{e d}^{V, L R}\right]_{13 j j}$ & -1.14 & -1.78 & 1.78 \\
\hline$\left[C_{e u}^{V, L L}\right]_{1311}$ & 2.28 & 3.57 & -3.56 \\
\hline$\left[C_{e u}^{V, L R}\right]_{1311}$ & 2.28 & 3.51 & -3.51 \\
\hline \multicolumn{4}{|l|}{$\left[\mathcal{C}_{e u}\right]_{1333}(\Lambda)=1$} \\
\hline$\left[C_{d e}^{V, L R}\right]_{j j 13}$ & - & 1.28 & -60.47 \\
\hline$\left[C_{e d}^{V, R R}\right]_{13 j j}$ & - & 1.25 & 12.50 \\
\hline$\left[C_{u e}^{V, L R}\right]_{1113}$ & - & -2.48 & 46.25 \\
\hline$\left[C_{e u}^{V, R R}\right]_{1311}$ & - & -2.62 & -25.80 \\
\hline \multicolumn{4}{|l|}{$\left[\mathcal{C}_{e d}\right]_{1333}(\Lambda)=1$} \\
\hline$\left[C_{d e}^{V, L R}\right]_{j j 13}$ & -1.14 & -1.76 & -1.75 \\
\hline$\left[C_{e d}^{V, R R}\right]_{13 j j}$ & -1.14 & -1.75 & -1.75 \\
\hline$\left[C_{u e}^{V, L R}\right]_{1113}$ & 2.28 & 3.48 & 3.47 \\
\hline$\left[C_{e u}^{V, R R}\right]_{1311}$ & 2.28 & 3.55 & 3.56 \\
\hline \multicolumn{4}{|l|}{$\left[\mathcal{C}_{q e}\right]_{1333}(\Lambda)=1$} \\
\hline$\left[C_{d e}^{V, L R}\right]_{j j 13}$ & -1.13 & -0.49 & -60.48 \\
\hline$\left[C_{e d}^{V, R R}\right]_{13 j j}$ & -1.13 & -0.51 & 11.62 \\
\hline$\left[C_{u e}^{V, L R}\right]_{1113}$ & 2.28 & 1.04 & 47.08 \\
\hline$\left[C_{e u}^{V, R R}\right]_{1311}$ & 2.27 & 0.96 & -23.86 \\
\hline
\end{tabular}

Table 14. The impact of semileptonic operators on the low energy semileptonic $\Delta F=(1,0)$ Wilson coefficients (in $10^{-9} \mathrm{TeV}^{-2}$ units) at $\mu_{\text {low }}=2 \mathrm{GeV}$ is shown. The second column refers to the values with only WET (QED+QCD) running, the third column refers to WET + SMEFT running due to gauge interactions, and the fourth column refers to the full WET+ SMEFT gauge + Yukawa running. Here the indices $j j=11$ or 22 and $\Lambda=1 \mathrm{TeV}$. The SMEFT Wilson coefficients are set to 1 (in $\mathrm{TeV}^{-2}$ units) at the high scale. 


\begin{tabular}{|c|c|c|c|}
\hline \multicolumn{4}{|c|}{$\bar{\ell}_{i} \gamma^{\mu} \ell_{j} \bar{q}_{k} \gamma_{\mu} q_{l}$} \\
\hline & $\Delta F=(0,0)$ & & $\Delta F=(1,0)$ \\
\hline 1111 & $(-1.37 \pm 1.15) \cdot 10^{0}$ & 1211 & $(1.64 \pm 0.62) \cdot 10^{-2}$ \\
\hline 1122 & $(6.94 \pm 1.68) \cdot 10^{0}$ & 1222 & $(1.63 \pm 0.62) \cdot 10^{-2}$ \\
\hline 1133 & $(-7.42 \pm 3.71) \cdot 10^{-1}$ & 1233 & $(2.42 \pm 0.91) \cdot 10^{-3}$ \\
\hline 2211 & $(8.77 \pm 6.07) \cdot 10^{1}$ & 1311 & $(3.99 \pm 1.03) \cdot 10^{0}$ \\
\hline 2222 & $(-6.32 \pm 1.46) \cdot 10^{0}$ & 1322 & $(7.48 \pm 2.74) \cdot 10^{-2}$ \\
\hline 2233 & $(2.85 \pm 0.62) \cdot 10^{0}$ & 1333 & $(8.64 \pm 1.73) \cdot 10^{-1}$ \\
\hline 3311 & $(-2.68 \pm 1.82) .10^{1}$ & 2311 & $(3.16 \pm 0.91) \cdot 10^{0}$ \\
\hline 3322 & $(-4.91 \pm 3.36) \cdot 10^{1}$ & 2322 & $(1.19 \pm 0.46) \cdot 10^{-1}$ \\
\hline 3333 & $(-0.19 \pm 1.36) \cdot 10^{0}$ & 2333 & $(6.93 \pm 1.64) \cdot 10^{-1}$ \\
\hline
\end{tabular}

Table 15. Allowed values of the Wilson coefficients $\left[c_{l q}^{(1)}\right]_{i j k l}$ based on the constraints discussed in the main text.

\begin{tabular}{|rrrc|}
\hline \multicolumn{4}{|c|}{$\bar{\ell}_{i} \gamma^{\mu} \tau^{a} \ell_{j} \bar{q}_{k} \gamma_{\mu} \tau^{a} q_{l}$} \\
\hline \multicolumn{3}{|c|}{$\Delta F=(0,0)$} & \multicolumn{1}{c|}{$\Delta F=(1,0)$} \\
\hline 1111 & $(1.84 \pm 3.44) \cdot 10^{-2}$ & 1211 & $(5.75 \pm 2.15) \cdot 10^{-3}$ \\
1122 & $(2.08 \pm 1.85) \cdot 10^{-1}$ & 1222 & $(5.75 \pm 2.14) \cdot 10^{-3}$ \\
1133 & $(-1.49 \pm 0.39) \cdot 10^{0}$ & 1233 & $(2.07 \pm 0.77) \cdot 10^{-3}$ \\
2211 & $(7.41 \pm 5.86) \cdot 10^{0}$ & 1311 & $(3.99 \pm 1.31) \cdot 10^{-2}$ \\
2222 & $(-2.17 \pm 1.76) \cdot 10^{-1}$ & 1322 & $(7.56 \pm 2.74) \cdot 10^{-2}$ \\
2233 & $(4.01 \pm 2.53) \cdot 10^{-1}$ & 1333 & $(6.86 \pm 1.43) \cdot 10^{-1}$ \\
3311 & $(8.73 \pm 5.04) \cdot 10^{-1}$ & 2311 & $(3.13 \pm 1.09) \cdot 10^{-2}$ \\
3322 & $(1.71 \pm 1.01) \cdot 10^{0}$ & 2322 & $(1.28 \pm 0.44) \cdot 10^{-1}$ \\
3333 & $(-0.10 \pm 1.07) \cdot 10^{0}$ & 2333 & $(5.40 \pm 1.30) \cdot 10^{-1}$ \\
\hline
\end{tabular}

Table 16. Same as in table 15, except for Wilson coefficient $\left[c_{l q}^{(3)}\right]_{i j k l}$. 


\begin{tabular}{|c|c|c|c|}
\hline \multicolumn{4}{|c|}{$\bar{\ell}_{i} \gamma^{\mu} \ell_{j} \bar{d}_{k} \gamma_{\mu} d_{l}$} \\
\hline & $\Delta F=(0,0)$ & & $\Delta F=(1,0)$ \\
\hline 1111 & $(-1.49 \pm 2.26) \cdot 10^{1}$ & 1211 & $(1.76 \pm 0.67) \cdot 10^{-2}$ \\
\hline 1122 & $(2.47 \pm 1.59) \cdot 10^{1}$ & 1222 & $(1.76 \pm 0.67) \cdot 10^{-2}$ \\
\hline 1133 & $(-4.09 \pm 1.63) \cdot 10^{1}$ & 1233 & $(2.24 \pm 0.84) \cdot 10^{-2}$ \\
\hline 2211 & $(-8.99 \pm 5.58) \cdot 10^{1}$ & 1311 & $(7.80 \pm 2.73) \cdot 10^{-2}$ \\
\hline 2222 & $(-7.04 \pm 2.16) .10^{1}$ & 1322 & $(6.94 \pm 2.59) \cdot 10^{-2}$ \\
\hline 2233 & $(4.34 \pm 2.12) \cdot 10^{1}$ & 1333 & $(7.77 \pm 1.94) \cdot 10^{0}$ \\
\hline 3311 & $(-0.22 \pm 6.83) \cdot 10^{1}$ & 2311 & $(6.36 \pm 2.25) \cdot 10^{-2}$ \\
\hline 3322 & $(-0.24 \pm 6.83) \cdot 10^{1}$ & 2322 & $(1.18 \pm 0.43) \cdot 10^{-1}$ \\
\hline 3333 & $(-1.54 \pm 6.89) \cdot 10^{1}$ & 2333 & $(6.29 \pm 1.65) \cdot 10^{0}$ \\
\hline
\end{tabular}

Table 17. Same as in table 15, except for Wilson coefficient $\left[c_{l d}\right]_{i j k l}$.

\begin{tabular}{|crrr|}
\hline \multicolumn{4}{|c|}{$\bar{\ell}_{i} \gamma^{\mu} \ell_{j} \bar{u}_{k} \gamma_{\mu} u_{l}$} \\
\hline \multicolumn{3}{|c|}{$\Delta F=(0,0)$} & \multicolumn{1}{c|}{$\Delta F=(1,0)$} \\
\hline 1111 & $(0.60 \pm 1.15) \cdot 10^{1}$ & 1211 & $(9.01 \pm 3.38) \cdot 10^{-3}$ \\
1122 & $(0.60 \pm 1.15) \cdot 10^{1}$ & 1222 & $(9.01 \pm 3.38) \cdot 10^{-3}$ \\
1133 & $(4.23 \pm 4.46) \cdot 10^{-1}$ & 1233 & $(2.53 \pm 0.95) \cdot 10^{-3}$ \\
2211 & $(4.02 \pm 2.80) \cdot 10^{1}$ & 1311 & $(8.28 \pm 2.89) \cdot 10^{-2}$ \\
2222 & $(4.05 \pm 2.80) \cdot 10^{1}$ & 1322 & $(3.42 \pm 0.84) \cdot 10^{0}$ \\
2233 & $(-3.70 \pm 0.93) \cdot 10^{0}$ & 1333 & $(9.54 \pm 1.88) \cdot 10^{-1}$ \\
3311 & $(-0.52 \pm 3.44) \cdot 10^{1}$ & 2311 & $(6.45 \pm 2.50) \cdot 10^{-2}$ \\
3322 & $(-0.48 \pm 3.44) \cdot 10^{1}$ & 2322 & $(2.72 \pm 0.74) \cdot 10^{0}$ \\
3333 & $(0.39 \pm 1.44) \cdot 10^{0}$ & 2333 & $(7.69 \pm 1.79) \cdot 10^{-1}$ \\
\hline
\end{tabular}

Table 18. Same as in table 15, except for Wilson coefficient $\left[c_{l u}\right]_{i j k l}$. 


\begin{tabular}{|c|c|c|c|}
\hline \multicolumn{4}{|c|}{$\bar{e}_{i} \gamma^{\mu} e_{j} \bar{d}_{k} \gamma_{\mu} d_{l}$} \\
\hline & $\Delta F=(0,0)$ & & $\Delta F=(1,0)$ \\
\hline 1111 & $(1.75 \pm 2.83) .10^{1}$ & 1211 & $(1.80 \pm 0.68) .10^{-2}$ \\
\hline 1122 & $(1.55 \pm 3.00) \cdot 10^{1}$ & 1222 & $(1.80 \pm 0.68) \cdot 10^{-2}$ \\
\hline 1133 & $(2.15 \pm 3.03) \cdot 10^{1}$ & 1233 & $(2.27 \pm 0.86) \cdot 10^{-2}$ \\
\hline 2211 & $(9.50 \pm 7.27) \cdot 10^{1}$ & 1311 & $(7.89 \pm 2.90) \cdot 10^{-2}$ \\
\hline 2222 & $(6.65 \pm 5.43) \cdot 10^{1}$ & 1322 & $(7.18 \pm 2.77) \cdot 10^{-2}$ \\
\hline 2233 & $(3.04 \pm 5.43) \cdot 10^{1}$ & 1333 & $(7.69 \pm 2.05) \cdot 10^{0}$ \\
\hline 3311 & $(-7.88 \pm 8.08) \cdot 10^{1}$ & 2311 & $(6.57 \pm 2.35) \cdot 10^{-2}$ \\
\hline 3322 & $(-7.92 \pm 8.08) \cdot 10^{1}$ & 2322 & $(1.19 \pm 0.46) \cdot 10^{-1}$ \\
\hline 3333 & $(-9.74 \pm 8.16) \cdot 10^{1}$ & 2333 & $(6.15 \pm 1.56) \cdot 10^{0}$ \\
\hline
\end{tabular}

Table 19. Same as in table 15, except for Wilson coefficient $\left[c_{e d}\right]_{i j k l}$.

\begin{tabular}{|cccr|}
\hline \multicolumn{3}{|c|}{$\bar{e}_{i} \gamma^{\mu} e_{j} \bar{u}_{k} \gamma_{\mu} u_{l}$} \\
\hline \multicolumn{3}{|c|}{$\Delta F=(0,0)$} & \multicolumn{1}{c|}{$\Delta F=(1,0)$} \\
\hline 1111 & $(-0.78 \pm 1.35) \cdot 10^{1}$ & 1211 & $(8.71 \pm 3.31) \cdot 10^{-3}$ \\
1122 & $(-0.78 \pm 1.35) \cdot 10^{1}$ & 1222 & $(8.71 \pm 3.31) \cdot 10^{-3}$ \\
1133 & $(2.63 \pm 5.32) \cdot 10^{-1}$ & 1233 & $(2.42 \pm 0.91) \cdot 10^{-3}$ \\
2211 & $(-4.43 \pm 3.51) \cdot 10^{1}$ & 1311 & $(7.73 \pm 2.61) \cdot 10^{-2}$ \\
2222 & $(-4.39 \pm 3.50) \cdot 10^{1}$ & 1322 & $(3.30 \pm 0.84) \cdot 10^{0}$ \\
2233 & $(2.06 \pm 1.30) \cdot 10^{0}$ & 1333 & $(9.41 \pm 1.84) \cdot 10^{-1}$ \\
3311 & $(4.26 \pm 4.02) \cdot 10^{1}$ & 2311 & $(6.39 \pm 2.18) \cdot 10^{-2}$ \\
3322 & $(4.31 \pm 4.01) \cdot 10^{1}$ & 2322 & $(2.65 \pm 0.66) \cdot 10^{0}$ \\
3333 & $(-1.44 \pm 1.57) \cdot 10^{0}$ & 2333 & $(7.76 \pm 1.67) \cdot 10^{-1}$ \\
\hline
\end{tabular}

Table 20. Same as in table 15, except for Wilson coefficient $\left[c_{e u}\right]_{i j k l}$.

\begin{tabular}{|c|c|c|c|}
\hline \multicolumn{4}{|c|}{$\bar{q}_{i} \gamma^{\mu} q_{j} \bar{e}_{k} \gamma_{\mu} e_{l}$} \\
\hline & $\Delta F=(0,0)$ & & $\Delta F=(1,0)$ \\
\hline 1111 & $(-1.98 \pm 2.76) \cdot 10^{1}$ & 1112 & $(1.82 \pm 0.68) \cdot 10^{-2}$ \\
\hline 2211 & $(-2.93 \pm 0.44) \cdot 10^{1}$ & 2212 & $(1.83 \pm 0.68) \cdot 10^{-2}$ \\
\hline 3311 & $(-2.86 \pm 5.26) \cdot 10^{-1}$ & 3312 & $(2.53 \pm 0.94) \cdot 10^{-3}$ \\
\hline 1122 & $(-8.69 \pm 7.11) \cdot 10^{1}$ & 1113 & $(8.26 \pm 2.96) \cdot 10^{-1}$ \\
\hline 2222 & $(5.22 \pm 4.87) \cdot 10^{0}$ & 2213 & $(6.90 \pm 2.68) \cdot 10^{-2}$ \\
\hline 3322 & $(-2.04 \pm 1.16) \cdot 10^{0}$ & 3313 & $(8.85 \pm 1.78) \cdot 10^{-1}$ \\
\hline 1133 & $(8.87 \pm 8.13) \cdot 10^{1}$ & 1123 & $(6.82 \pm 2.47) \cdot 10^{-1}$ \\
\hline 2233 & $(7.40 \pm 7.39) \cdot 10^{1}$ & 2223 & $(1.16 \pm 0.44) \cdot 10^{-1}$ \\
\hline 3333 & $(1.32 \pm 1.52) \cdot 10^{0}$ & 3323 & $(7.30 \pm 1.67) \cdot 10^{-1}$ \\
\hline
\end{tabular}

Table 21. Same as in table 15, except for Wilson coefficient $\left[c_{q e}\right]_{i j k l}$. 
Open Access. This article is distributed under the terms of the Creative Commons Attribution License (CC-BY 4.0), which permits any use, distribution and reproduction in any medium, provided the original author(s) and source are credited.

\section{References}

[1] B. Grzadkowski, M. Iskrzynski, M. Misiak and J. Rosiek, Dimension-Six Terms in the Standard Model Lagrangian, JHEP 10 (2010) 085 [arXiv:1008.4884] [INSPIRE].

[2] W. Buchmüller and D. Wyler, Effective Lagrangian Analysis of New Interactions and Flavor Conservation, Nucl. Phys. B 268 (1986) 621 [INSPIRE].

[3] A. Buras, Gauge Theory of Weak Decays, Cambridge University Press (2020).

[4] B. Bhattacharya, A. Datta, D. London and S. Shivashankara, Simultaneous Explanation of the $R_{K}$ and $R\left(D^{(*)}\right)$ Puzzles, Phys. Lett. B $\mathbf{7 4 2}$ (2015) 370 [arXiv:1412.7164] [InSPIRE].

[5] B. Capdevila, A. Crivellin, S. Descotes-Genon, L. Hofer and J. Matias, Searching for New Physics with $b \rightarrow s \tau^{+} \tau^{-}$processes, Phys. Rev. Lett. 120 (2018) 181802 [arXiv:1712.01919] [INSPIRE].

[6] A.J. Buras, J. Girrbach-Noe, C. Niehoff and D.M. Straub, $B \rightarrow K^{(*)} \nu \bar{\nu}$ decays in the Standard Model and beyond, JHEP 02 (2015) 184 [arXiv:1409.4557] [INSPIRE].

[7] F. Feruglio, P. Paradisi and A. Pattori, On the Importance of Electroweak Corrections for B Anomalies, JHEP 09 (2017) 061 [arXiv:1705.00929] [INSPIRE].

[8] C. Cornella, F. Feruglio and P. Paradisi, Low-energy Effects of Lepton Flavour Universality Violation, JHEP 11 (2018) 012 [arXiv: 1803.00945] [INSPIRE].

[9] J. Aebischer, A.J. Buras and J. Kumar, Another SMEFT story: $Z^{\prime}$ facing new results on $\epsilon^{\prime} / \epsilon$, $\Delta M_{K}$ and $K \rightarrow \pi \nu \bar{\nu}, J H E P 12$ (2020) 097 [arXiv: 2006.01138] [INSPIRE].

[10] J. Aebischer and J. Kumar, Flavour violating effects of Yukawa running in SMEFT, JHEP 09 (2020) 187 [arXiv: 2005.12283] [INSPIRE].

[11] J. Aebischer, C. Bobeth, A.J. Buras and J. Kumar, SMEFT ATLAS of $\Delta F=2$ transitions, JHEP 12 (2020) 187 [arXiv: 2009.07276] [INSPIRE].

[12] S. Bruggisser, R. Schäfer, D. van Dyk and S. Westhoff, The Flavor of UV Physics, JHEP 05 (2021) 257 [arXiv: 2101.07273] [INSPIRE].

[13] R. Alonso, E.E. Jenkins, A.V. Manohar and M. Trott, Renormalization Group Evolution of the Standard Model Dimension Six Operators III: Gauge Coupling Dependence and Phenomenology, JHEP 04 (2014) 159 [arXiv: 1312.2014] [INSPIRE].

[14] E.E. Jenkins, A.V. Manohar and M. Trott, Renormalization Group Evolution of the Standard Model Dimension Six Operators II: Yukawa Dependence, JHEP 01 (2014) 035 [arXiv: 1310.4838] [INSPIRE].

[15] E.E. Jenkins, A.V. Manohar and M. Trott, Renormalization Group Evolution of the Standard Model Dimension Six Operators I: Formalism and lambda Dependence, JHEP 10 (2013) 087 [arXiv: 1308.2627] [INSPIRE].

[16] E.E. Jenkins, A.V. Manohar and P. Stoffer, Low-Energy Effective Field Theory below the Electroweak Scale: Anomalous Dimensions, JHEP 01 (2018) 084 [arXiv:1711.05270] [INSPIRE]. 
[17] J. Aebischer, M. Fael, C. Greub and J. Virto, B physics Beyond the Standard Model at One Loop: Complete Renormalization Group Evolution below the Electroweak Scale, JHEP 09 (2017) 158 [arXiv:1704.06639] [INSPIRE].

[18] M. Chala and A. Titov, One-loop running of dimension-six Higgs-neutrino operators and implications of a large neutrino dipole moment, JHEP 09 (2020) 188 [arXiv:2006.14596] [INSPIRE].

[19] A. Datta, J. Kumar, H. Liu and D. Marfatia, Anomalous dimensions from gauge couplings in SMEFT with right-handed neutrinos, JHEP 02 (2021) 015 [arXiv:2010.12109] [INSPIRE].

[20] A. Datta, J. Kumar, H. Liu and D. Marfatia, Anomalous dimensions from Yukawa couplings in SMNEFT: four-fermion operators, JHEP 05 (2021) 037 [arXiv: 2103.04441] [INSPIRE].

[21] J. Aebischer, J. Kumar and D.M. Straub, Wilson: a Python package for the running and matching of Wilson coefficients above and below the electroweak scale, Eur. Phys. J. C 78 (2018) 1026 [arXiv:1804.05033] [INSPIRE].

[22] A. Celis, J. Fuentes-Martin, A. Vicente and J. Virto, DsixTools: The Standard Model Effective Field Theory Toolkit, Eur. Phys. J. C 77 (2017) 405 [arXiv: 1704.04504] [InSPIRE].

[23] J. Fuentes-Martin, P. Ruiz-Femenia, A. Vicente and J. Virto, DsixTools 2.0: The Effective Field Theory Toolkit, Eur. Phys. J. C 81 (2021) 167 [arXiv:2010.16341] [InSPIRE].

[24] A.J. Buras and M. Jung, Analytic inclusion of the scale dependence of the anomalous dimension matrix in Standard Model Effective Theory, JHEP 06 (2018) 067 [arXiv: 1804.05852] [INSPIRE].

[25] D.M. Straub, flavio: a Python package for flavour and precision phenomenology in the Standard Model and beyond, arXiv:1810.08132 [INSPIRE].

[26] I. Brivio, Y. Jiang and M. Trott, The SMEFTsim package, theory and tools, JHEP 12 (2017) 070 [arXiv: 1709. 06492] [INSPIRE].

[27] A. Dedes, M. Paraskevas, J. Rosiek, K. Suxho and L. Trifyllis, SmeftFR - Feynman rules generator for the Standard Model Effective Field Theory, Comput. Phys. Commun. 247 (2020) 106931 [arXiv: 1904.03204] [INSPIRE].

[28] J. Aebischer, M. Fael, A. Lenz, M. Spannowsky and J. Virto, eds., Computing Tools for the SMEFT, (2019), https://indico.cern.ch/event/787665/.

[29] M. Endo, S. Mishima and D. Ueda, Revisiting electroweak radiative corrections to $b \rightarrow$ sll in SMEFT, JHEP 05 (2021) 050 [arXiv: 2012.06197] [INSPIRE].

[30] L. Alasfar, A. Azatov, J. de Blas, A. Paul and M. Valli, $B$ anomalies under the lens of electroweak precision, JHEP 12 (2020) 016 [arXiv:2007.04400] [INSPIRE].

[31] A. Celis, J. Fuentes-Martin, A. Vicente and J. Virto, Gauge-invariant implications of the LHCb measurements on lepton-flavor nonuniversality, Phys. Rev. D 96 (2017) 035026 [arXiv: 1704.05672] [INSPIRE].

[32] J. Kumar, D. London and R. Watanabe, Combined Explanations of the $b \rightarrow s \mu^{+} \mu^{-}$and $b \rightarrow c \tau^{-} \bar{\nu}$ Anomalies: a General Model Analysis, Phys. Rev. D 99 (2019) 015007 [arXiv: 1806.07403] [INSPIRE].

[33] C. Bobeth and A.J. Buras, Leptoquarks meet $\varepsilon^{\prime} / \varepsilon$ and rare Kaon processes, JHEP 02 (2018) 101 [arXiv: 1712.01295] [INSPIRE]. 
[34] D. Buttazzo, A. Greljo, G. Isidori and D. Marzocca, B-physics anomalies: a guide to combined explanations, JHEP 11 (2017) 044 [arXiv: 1706. 07808] [INSPIRE].

[35] L. Calibbi, A. Crivellin and T. Ota, Effective Field Theory Approach to $b \rightarrow$ sl( $^{\left({ }^{\prime}\right)}$, $B \rightarrow K^{(*)} \nu \bar{\nu}$ and $B \rightarrow D^{(*)} \tau \nu$ with Third Generation Couplings, Phys. Rev. Lett. 115 (2015) 181801 [arXiv: 1506.02661] [INSPIRE].

[36] R. Alonso, B. Grinstein and J. Martin Camalich, Lepton universality violation and lepton flavor conservation in B-meson decays, JHEP 10 (2015) 184 [arXiv: 1505. 05164] [INSPIRE].

[37] S. Dawson and P.P. Giardino, Electroweak and QCD corrections to $Z$ and $W$ pole observables in the standard model EFT, Phys. Rev. D 101 (2020) 013001 [arXiv:1909.02000] [INSPIRE].

[38] F. Feruglio, P. Paradisi and A. Pattori, Revisiting Lepton Flavor Universality in B Decays, Phys. Rev. Lett. 118 (2017) 011801 [arXiv:1606.00524] [INSPIRE].

[39] J. Aebischer, J. Kumar, P. Stangl and D.M. Straub, A Global Likelihood for Precision Constraints and Flavour Anomalies, Eur. Phys. J. C 79 (2019) 509 [arXiv:1810.07698] [INSPIRE].

[40] J. Aebischer et al., WCxf: an exchange format for Wilson coefficients beyond the Standard Model, Comput. Phys. Commun. 232 (2018) 71 [arXiv:1712.05298] [InSPIRE].

[41] S. Descotes-Genon, A. Falkowski, M. Fedele, M. González-Alonso and J. Virto, The CKM parameters in the SMEFT, JHEP 05 (2019) 172 [arXiv:1812.08163] [INSPIRE].

[42] A. Falkowski and F. Riva, Model-independent precision constraints on dimension-6 operators, JHEP 02 (2015) 039 [arXiv: 1411.0669] [InSPIRE].

[43] J. Ellis, V. Sanz and T. You, The Effective Standard Model after LHC Run I, JHEP 03 (2015) 157 [arXiv: 1410.7703] [INSPIRE].

[44] A. Efrati, A. Falkowski and Y. Soreq, Electroweak constraints on flavorful effective theories, JHEP 07 (2015) 018 [arXiv: 1503. 07872] [INSPIRE].

[45] L. Berthier and M. Trott, Towards consistent Electroweak Precision Data constraints in the SMEFT, JHEP 05 (2015) 024 [arXiv:1502.02570] [INSPIRE].

[46] J. Ellis, C.W. Murphy, V. Sanz and T. You, Updated Global SMEFT Fit to Higgs, Diboson and Electroweak Data, JHEP 06 (2018) 146 [arXiv:1803.03252] [INSPIRE].

[47] V. Bresó-Pla, A. Falkowski and M. González-Alonso, $A_{F B}$ in the SMEFT: precision Z physics at the LHC, JHEP 08 (2021) 021 [arXiv:2103.12074] [INSPIRE].

[48] A. Falkowski and D. Straub, Flavourful SMEFT likelihood for Higgs and electroweak data, JHEP 04 (2020) 066 [arXiv: 1911.07866] [INSPIRE].

[49] I. Brivio and M. Trott, The Standard Model as an Effective Field Theory, Phys. Rept. 793 (2019) 1 [arXiv: 1706. 08945] [inSPIRE].

[50] S. Dawson and P.P. Giardino, Higgs decays to $Z Z$ and $Z \gamma$ in the standard model effective field theory: An NLO analysis, Phys. Rev. D 97 (2018) 093003 [arXiv:1801.01136] [INSPIRE].

[51] Anisha, S. Das Bakshi, J. Chakrabortty and S.K. Patra, Connecting electroweak-scale observables to BSM physics through EFT and Bayesian statistics, Phys. Rev. D 103 (2021) 076007 [arXiv: 2010.04088] [INSPIRE].

[52] A. Crivellin, S. Davidson, G.M. Pruna and A. Signer, Renormalisation-group improved analysis of $\mu \rightarrow e$ processes in a systematic effective-field-theory approach, JHEP 05 (2017) 117 [arXiv: 1702.03020$]$ [INSPIRE]. 
[53] V. Cirigliano, K. Fuyuto, C. Lee, E. Mereghetti and B. Yan, Charged Lepton Flavor Violation at the EIC, JHEP 03 (2021) 256 [arXiv:2102.06176] [INSPIRE].

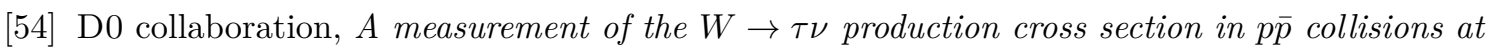
$\sqrt{s}=1.8 \mathrm{TeV}$, Phys. Rev. Lett. 84 (2000) 5710 [hep-ex/9912065] [inSPIRE].

[55] LHCb collaboration, Measurement of forward $W \rightarrow e \nu$ production in pp collisions at $\sqrt{s}=8 \mathrm{TeV}$, JHEP 10 (2016) 030 [arXiv:1608.01484] [INSPIRE].

[56] ALEPH, DELPHI, L3, OPAL and LEP EleCtroweAK collaborations, Electroweak Measurements in Electron-Positron Collisions at W-Boson-Pair Energies at LEP, Phys. Rept. 532 (2013) 119 [arXiv:1302.3415] [INSPIRE].

[57] CDF and D0 collaborations, Combination of CDF and D0 W-Boson Mass Measurements, Phys. Rev. D 88 (2013) 052018 [arXiv:1307.7627] [INSPIRE].

[58] ATLAS collaboration, Measurement of the $W$-boson mass in pp collisions at $\sqrt{s}=7 \mathrm{TeV}$ with the ATLAS detector, Eur. Phys. J. C 78 (2018) 110 [Erratum ibid. 78 (2018) 898] [arXiv: 1701.07240] [INSPIRE].

[59] SLD collaboration, First direct measurement of the parity violating coupling of the $Z^{0}$ to the $s$ quark, Phys. Rev. Lett. 85 (2000) 5059 [hep-ex/0006019] [InSPIRE].

[60] ALEPH, DELPHI, L3, OPAL, SLD, LEP Electroweak Working Group, SLD Electroweak Group and SLD Heavy Flavour Group collaborations, Precision electroweak measurements on the $Z$ resonance, Phys. Rept. 427 (2006) 257 [hep-ex/0509008] [INSPIRE].

[61] ATLAS collaboration, Test of the universality of $\tau$ and $\mu$ lepton couplings in $W$-boson decays with the ATLAS detector, Nature Phys. 17 (2021) 813 [arXiv:2007.14040] [INSPIRE].

[62] R. Coy, M. Frigerio, F. Mescia and O. Sumensari, New physics in $b \rightarrow$ sll transitions at one loop, Eur. Phys. J. C 80 (2020) 52 [arXiv: 1909.08567] [InSPIRE].

[63] J. Aebischer, A. Crivellin, M. Fael and C. Greub, Matching of gauge invariant dimension-six operators for $b \rightarrow s$ and $b \rightarrow c$ transitions, JHEP 05 (2016) 037 [arXiv:1512.02830] [INSPIRE].

[64] G. Hiller and F. Krüger, More model-independent analysis of $b \rightarrow s$ processes, Phys. Rev. D 69 (2004) 074020 [hep-ph/0310219] [INSPIRE].

[65] LHCb collaboration, Test of lepton universality using $B^{+} \rightarrow K^{+} \ell^{+} \ell^{-}$decays, Phys. Rev. Lett. 113 (2014) 151601 [arXiv: 1406.6482] [INSPIRE].

[66] LHCb collaboration, Search for lepton-universality violation in $B^{+} \rightarrow K^{+} \ell^{+} \ell^{-}$decays, Phys. Rev. Lett. 122 (2019) 191801 [arXiv: 1903.09252] [INSPIRE].

[67] BELLE collaboration, Test of lepton flavor universality and search for lepton flavor violation in $B \rightarrow$ Kll decays, JHEP 03 (2021) 105 [arXiv: 1908.01848] [INSPIRE].

[68] LHCb collaboration, Test of lepton universality in beauty-quark decays, arXiv:2103.11769 [INSPIRE].

[69] LHCb collaboration, Test of lepton universality with $B^{0} \rightarrow K^{* 0} \ell^{+} \ell^{-}$decays, JHEP 08 (2017) 055 [arXiv: 1705. 05802] [INSPIRE].

[70] BeLLe collaboration, Test of Lepton-Flavor Universality in $B \rightarrow K^{*} \ell^{+} \ell^{-}$Decays at Belle, Phys. Rev. Lett. 126 (2021) 161801 [arXiv:1904.02440] [INSPIRE]. 
[71] Belle collaboration, Lepton-Flavor-Dependent Angular Analysis of $B \rightarrow K^{*} \ell^{+} \ell^{-}$, Phys. Rev. Lett. 118 (2017) 111801 [arXiv: 1612.05014] [INSPIRE].

[72] B. Capdevila, S. Descotes-Genon, J. Matias and J. Virto, Assessing lepton-flavour non-universality from $B \rightarrow K^{*} \ell \ell$ angular analyses, JHEP 10 (2016) 075 [arXiv:1605.03156] [INSPIRE].

[73] A. Datta, J. Kumar and D. London, The B anomalies and new physics in $b \rightarrow s e^{+} e^{-}$, Phys. Lett. B 797 (2019) 134858 [arXiv:1903.10086] [INSPIRE].

[74] W. Altmannshofer and P. Stangl, New physics in rare B decays after Moriond 2021, Eur. Phys. J. C 81 (2021) 952 [arXiv:2103.13370] [INSPIRE].

[75] J.C. Hardy and I.S. Towner, Superallowed $0^{+} \rightarrow 0^{+}$nuclear $\beta$ decays: 2014 critical survey, with precise results for $V_{u d}$ and CKM unitarity, Phys. Rev. C 91 (2015) 025501 [arXiv: 1411.5987] [INSPIRE].

[76] Particle Data Group collaboration, Review of Particle Physics, Chin. Phys. C 38 (2014) 090001 [INSPIRE].

[77] PIENu collaboration, Improved Measurement of the $\pi \rightarrow e \nu$ Branching Ratio, Phys. Rev. Lett. 115 (2015) 071801 [arXiv:1506.05845] [INSPIRE].

[78] Belle collaboration, Study of $\tau^{-} \rightarrow K_{S} \pi^{-} \nu_{\tau}$ decay at Belle, Phys. Lett. B 654 (2007) 65 [arXiv:0706.2231] [INSPIRE].

[79] A. Pich, Precision Tau Physics, Prog. Part. Nucl. Phys. 75 (2014) 41 [arXiv:1310.7922] [INSPIRE].

[80] S. Gonzàlez-Solís, A. Miranda, J. Rendón and P. Roig, Exclusive hadronic tau decays as probes of non-SM interactions, Phys. Lett. B 804 (2020) 135371 [arXiv:1912.08725] [InSPIRE].

[81] E.E. Jenkins, A.V. Manohar and P. Stoffer, Low-Energy Effective Field Theory below the Electroweak Scale: Operators and Matching, JHEP 03 (2018) 016 [arXiv:1709.04486] [INSPIRE].

[82] ATLAS collaboration, Search for the lepton flavor violating decay $Z \rightarrow e \mu$ in pp collisions at $\sqrt{s}=8 \mathrm{TeV}$ with the ATLAS detector, Phys. Rev. D 90 (2014) 072010 [arXiv:1408.5774] [INSPIRE].

[83] ATLAS collaboration, Search for lepton-flavor-violation in Z-boson decays with $\tau$-leptons with the ATLAS detector, arXiv:2105.12491 [INSPIRE].

[84] K. Hayasaka et al., Search for Lepton Flavor Violating Tau Decays into Three Leptons with 719 Million Produced Tau+Tau- Pairs, Phys. Lett. B 687 (2010) 139 [arXiv:1001.3221] [INSPIRE].

[85] SINDRUM collaboration, Search for the Decay $\mu^{+} \rightarrow e^{+} e^{+} e^{-}$, Nucl. Phys. B 299 (1988) 1 [INSPIRE].

[86] Particle Data Group collaboration, Review of Particle Physics, Chin. Phys. C 40 (2016) 100001 [INSPIRE].

[87] L. Calibbi, X. Marcano and J. Roy, Z lepton flavour violation as a probe for new physics at future $e^{+} e^{-}$colliders, Eur. Phys. J. C 81 (2021) 1054 [arXiv:2107.10273] [INSPIRE]. 